\title{
Subharmonic Solutions in Reversible Non-Autonomous Differential Equations
}

\author{
Izuchukwu Eze*, Carlos García-Azpeitiał Wieslaw Krawcewicz ${ }^{\ddagger}$ and Yanli Lv ${ }^{\S}$
}

\begin{abstract}
We study the existence of subharmonic solutions in the system $\ddot{u}(t)=f(t, u(t))$, where $u(t) \in \mathbb{R}^{k}$ and $f$ is an even and $p$-periodic function in time. Under some additional symmetry conditions on the function $f$, the problem of finding $m p$-periodic solutions can be reformulated in a functional space as a $\Gamma \times \mathbb{Z}_{2} \times D_{m}$-equivariant equation, where the group $\Gamma \times \mathbb{Z}_{2}$ acts on the space $\mathbb{R}^{k}$ and $D_{m}$ acts on $u(t)$ by time-shifts and reflection. We apply Brouwer equivariant degree to prove the existence of an infinite number of subharmonic solutions for the function $f$ that satisfies additional hypothesis on linear behavior near zero and the Nagumo condition at infinity. We also discuss the bifurcation of subharmonic solutions when the system depends on an extra parameter.
\end{abstract}

\section{Introduction}

In this paper we study the existence of subharmonic solutions of the system

$$
\ddot{u}(t)=f(t, u(t)), \quad u(t) \in \mathbb{R}^{k},
$$

where $f: \mathbb{R} \times \mathbb{R}^{k} \rightarrow \mathbb{R}^{k}$ is a continuous function satisfying the following conditions:

$\left(A_{1}\right) \quad$ For all $t \in \mathbb{R}$ and $x \in \mathbb{R}^{k}$ we have $f(t+2 \pi, x)=f(t, x)$;

$\left(A_{2}\right) \quad$ For all $t \in \mathbb{R}$ and $x \in \mathbb{R}^{k}$ we have $f(-t, x)=f(t, x)$;

$\left(A_{3}\right) \quad$ For all $t \in \mathbb{R}$ and $x \in \mathbb{R}^{k}$ we have $f(t,-x)=-f(t, x)$.

Notice that in condition $\left(A_{1}\right)$, one could consider $f(t, x)$ being $p$-periodic with respect to $t$, however by rescaling the time $t$, one can always arrive to a $2 \pi$-periodic function.

The problem of finding multiple subharmonic solutions to (11), especially in the case of Hamiltonian systems of the type

$$
\ddot{u}+\nabla F(t, u)=h(t)
$$

attracted a lot of attention. Let us mention several contributions, beginning with the classical work [3] and followed by the works [7, 21], with numerous other articles that were devoted to this topic (see [1, 6, 8, [10, 11, 12, 15, 17, 18, 19, 20, 22, 23, 24, 25, 26, 27, 29]). It

\footnotetext{
*Department of Mathematical Sciences the University of Texas at Dallas Richardson, 75080 USA, amos.eze@utdallas.edu

†Depto. Matemáticas y Mecánica IIMAS, Universidad Nacional Autónoma de México, Apdo. Postal 20-726, 01000 Ciudad de México, México, and Department of Mathematics, Xiangnan University, 889 Chen Zhou Da Dao, Chenzhou, Hunan 423000, China. cgazpe@mym.iimas.unam.mx

$¥$ Applied Mathematics Center at Guangzhou University, Guangzhou, 510006 China, and Department of Mathematical Sciences the University of Texas at Dallas Richardson, 75080 USA. wieslaw@utallas.edu

$\S$ Department of Mathematical Sciences the China Three Gorges University, Yichang, China , rebecca_utd@aliyun.com
} 
should be pointed out that variational structure of the system (2) seems to play crucial role for the application of the topological and geometric methods. Regarding the degree theory (cf. [13, 16]), it has been successfully applied to non-Hamiltonian systems in [4, 9]) (see also [5, 14, 28, 30]).

Conditions $\left(A_{1}\right)-\left(A_{3}\right)$ express the symmetric properties of the equation (1). Indeed, finding $2 \pi m$-periodic solutions to (1) leads to an operator which is $D_{m} \times \mathbb{Z}_{2}$-equivariant. Notice that the $\mathbb{Z}_{2}$-action allows us to make a distinction between constant and non-constant solutions. We do not require that $f$ is of a gradient-type or has any differentiability properties, except for the existence of the linearization at 0 .

Since problem (1) leads naturally to a $D_{m} \times \mathbb{Z}_{2}$-equivariant equation in functional spaces, one should ask a question: what would be the impact of additional (geometric) symmetries of equation (11) on the existence and multiplicity of subharmonic solutions? Therefore, it is natural to assume that the system (1) has additional symmetries represented by a group $\Gamma$. In this paper, we assume that $\Gamma$ is a finite group acting on vectors in $\mathbb{R}^{k}$ by permuting their coordinates (see assumption $\left(A_{4}\right)$ ), i.e. the functional equation has the symmetries

$$
G:=\Gamma \times D_{m} \times \mathbb{Z}_{2} .
$$

We use Brouwer G-equivariant degree to establish the existence and multiplicity of subharmonic $2 \pi m$-periodic solutions to (1). We make some additional assumptions in order to illustrate an application of the Brouwer equivariant degree to this systems of differential equations. First we assume (see the assumption $\left(A_{5}\right)$ ) that the linearization at 0 exists and is non-degenerate. We also impose on $f$ the Nagumo growth condition, which implies the existence of a priori bounds on periodic solutions to (1).

We explore in detail two cases of systems of equation: (a) non-symmetric (with $\Gamma$ being trivial), and (b) with additional symmetries $\Gamma=D_{3}$ and $\Gamma=D_{5}$. The group $D_{3}$ is the simplest non-abelian group, but it already makes a significant impact on the existence of multiple subharmonic solutions. Since the computations of Brouwer $G$-equivariant degree can be technically challenging, in order to overcome these difficulties we use the equivariant degree package EquiDeg for GAP programming, which was created by Hao-Pin $\mathrm{Wu}$ and is available from https://github.com/psistwu/GAP-equideg

As the assumption $\left(A_{3}\right)$ implies that $f(t, 0)=0$, so (1) admits the (trivial) solution $u(t)=$ 0 . It is interesting to study a parametrized by $\alpha$ modification of the system (11) (see system (28)) for which the existence of non-constant branches of subharmonic $2 \pi m$-periodic solutions bifurcating from 0 can be analyzed. We apply the Brouwer $G$-equivariant degree method to study the symmetric bifurcation problem for (28). We establish the existence of multiple branches of subharmonic solutions emerging from the trivial solutions as the parameter $\alpha$ crosses a critical value. Theoretical results are illustrated by an example involving concrete symmetries for the system (28).

\section{Reversible Non-Autonomous Differential Equations}

We are interested in studying the existence of the so-called subharmonic periodic solutions to (1), i.e. in finding non-constant solutions, which for some integer $m \geq 3$ satisfy

$$
u(t)=u(t+2 \pi m), \quad \dot{u}(t)=\dot{u}(t+2 \pi m) .
$$

We also consider a subgroup $\Gamma \leq S_{k}$ acting on $V:=\mathbb{R}^{k}$ by permuting the coordinates of vectors $x=\left(x_{1}, x_{2}, \ldots, x_{k}\right)^{T} \in \mathbb{R}^{\bar{k}}$, i.e. for $\sigma \in S_{k}$

$$
\sigma x=\sigma\left(x_{1}, x_{2}, \ldots, x_{k}\right)^{T}:=\left(x_{\sigma(1)}, x_{\sigma(2)}, \ldots, x_{\sigma(k)}\right)^{T} .
$$


Clearly, the space $V:=\mathbb{R}^{k}$ equipped with this $\Gamma$-action is an orthogonal $\Gamma$-representation. As it is also of our interest to study the impact of the symmetries $\Gamma$ on the existence of subharmonic solutions to (1), we introduce the following condition:

$\left(A_{4}\right) \quad$ For all $t \in \mathbb{R}, x \in \mathbb{R}^{k}$ and $\sigma \in \Gamma$, we have $f(t, \sigma x)=\sigma f(t, x)$.

The condition $\left(A_{4}\right)$ implies that $f$ is $\Gamma$-equivariant, i.e. the system (1) admits $\Gamma$-symmetries.

\subsection{Reformulation of (11) in Functional Spaces}

Consider the Banach space $\mathbb{F}:=C_{2 \pi m}(\mathbb{R}, V)$ of all $2 \pi m$-periodic continuous $V$-valued functions with the usual sup-norm

$$
\|\varphi\|_{\infty}:=\max _{t \in \mathbb{R}}|\varphi(t)|, \varphi \in \mathbb{F}
$$

and denote by $\mathbb{E}:=C_{2 \pi m}^{2}(\mathbb{R}, V)$ the Banach space of all $2 \pi m$-periodic $C^{2}$-differentiable $V$ valued functions with the norm $\|\cdot\|:=\|\cdot\|_{2, \infty}$ given by

$$
\|u\|=\|u\|_{2, \infty}:=\max \left\{\|u\|_{\infty},\|\dot{u}\|_{\infty},\|\ddot{u}\|_{\infty}\right\}, \quad u \in \mathbb{E} .
$$

Notice that the natural injection operator $\mathfrak{j}: \mathbb{E} \rightarrow \mathbb{F},(\mathfrak{j}(u))(t):=u(t), t \in \mathbb{R}$, is a compact linear operator. We define the operator $L: \mathbb{E} \rightarrow \mathbb{F}$ by $L(u)(t):=\ddot{u}(t)-u(t), u \in \mathbb{E}$, and the continuous map $N_{f}: \mathbb{F} \rightarrow \mathbb{F}$ by $N_{f}(\varphi)(t)=f(t, \varphi(t)), \varphi \in \mathbb{F}$. Then, the system (1) is equivalent to the following operator equation

$$
L u=N_{f}(\mathfrak{j}(u))-\mathfrak{j}(u), \quad u \in \mathbb{E} .
$$

Since the operator $L$ is an isomorphism, we can rewrite (6) as

$$
u=L^{-1}\left(N_{f}(\mathfrak{j}(u))-\mathfrak{j}(u)\right), \quad u \in \mathbb{E} .
$$

Define the map $\mathscr{F}: \mathbb{E} \rightarrow \mathbb{E}$, by

$$
\mathscr{F}(u):=u-L^{-1}\left(N_{f}(\mathfrak{j}(u))-\mathfrak{j}(u)\right), \quad u \in \mathbb{E} .
$$

Then $u \in \mathbb{E}$ is a solution to (1) if and only if

$$
\mathscr{F}(u)=0 .
$$

One can easily observe that, by compactness of $\mathfrak{j}$, the map $\mathscr{F}$ is a completely continuous field on $\mathbb{E}$.

Obviously, by the condition $\left(A_{3}\right)$, we have $f(t, 0)=0$ for all $t \in \mathbb{R}$, thus $\mathscr{F}(0)=0$, i.e. the zero function is the trivial solution to (1). In what follows we are interested in finding non-trivial (i.e. non-constant) $2 \pi m$-periodic solutions to (1). The group $G:=\Gamma \times D_{m} \times \mathbb{Z}_{2}$ acts on the space $\mathbb{E}$ by

$$
\begin{aligned}
\left(\sigma, \gamma^{j}, \pm 1\right) u(t) & := \pm \sigma u(t+2 \pi j), \quad j=0,1, \ldots, m-1, \quad \sigma \in \Gamma, \gamma=e^{\frac{i 2 \pi}{m}} \\
(\sigma, \kappa, \pm 1) u(t) & := \pm \sigma u(-t), \quad t \in \mathbb{R}, \quad u \in \mathbb{E}
\end{aligned}
$$

thus $\mathbb{E}$ is an isometric Banach $G$-representation. One can easily verify that the properties $\left(A_{1}\right)-\left(A_{4}\right)$ imply that $\mathscr{F}$ is $G$-equivariant. 


\section{$2.2 G$-Isotypic Decomposition of $\mathbb{E}$}

Actually, $\mathbb{E}$ is an isometric Banach $\Gamma \times O(2) \times \mathbb{Z}_{2}$-representation, with $O(2)$-action given by

$$
e^{i \theta} u(t)=u(t+\theta m), \quad \kappa u(t)=u(-t), \quad u \in \mathbb{E},
$$

and $\Gamma$-action given by $(\gamma u)(t)=\gamma u(t), \gamma \in \Gamma, t \in \mathbb{R}$. Using the $\Gamma \times O(2) \times \mathbb{Z}_{2}$-action on $\mathbb{E}$, one can easily recognize the $\Gamma \times D_{m} \times \mathbb{Z}_{2}$-isotypic decomposition of $\mathbb{E}$. Indeed, by using the usual Fourier series expansions of functions $u \in \mathbb{E}$, we have the following $\Gamma \times O(2) \times \mathbb{Z}_{2}$-isotypic decomposition of $\mathbb{E}$

$$
\mathbb{E}=\overline{\bigoplus_{j=0}^{\infty} \bigoplus_{l=0}^{\mathfrak{r}} \mathbb{V}_{j, l},}
$$

where

$$
\mathbb{V}_{j, l}=\left\{u \in \mathbb{E}: u(t)=\cos (j t / m) a+\sin (j t / m) b, a, b \in V_{l}\right\}
$$

and

$$
V=V_{0} \oplus V_{1} \oplus \cdots \oplus V_{\mathfrak{r}},
$$

is a $\Gamma$-isotypic decomposition of $V$, with the component $V_{l}$ being modeled on the $\Gamma$-irreducible representation $\mathcal{U}_{l}, 0 \leq l \leq \mathfrak{r}$.

Proposition 2.1. For $j>0$, the $\Gamma \times O(2) \times \mathbb{Z}_{2}$-invariant subspace $\mathbb{V}_{j, l}$ can be identified with the complexification $V_{l}^{c}:=V_{l} \oplus i V_{l}$ of $V_{l}$, on which $O(2)$ acts by

$$
e^{i \theta}(a+i b):=e^{-i j \theta} \cdot(a+i b), \quad \kappa(a+i b)=a-i b, \quad a, b \in V_{l},
$$

where '.' stands for complex multiplication.

Proof. Define the real isomorphism $\psi_{j}: V_{l}^{c} \rightarrow \mathbb{V}_{j, l}$ by $\psi(a+i b)(t)=\cos (j t / m) a+\sin (j t / m) b$, where $a, b \in V_{l}$. Then for $\mathbf{z}:=a+i b$ we have

$$
\begin{aligned}
\psi_{j}\left(e^{i \theta}(\mathbf{z})\right) & =\psi_{j}\left(e^{i \theta}(a+i b)\right) \\
& =\psi_{j}(\cos (j \theta) a+\sin (j \theta) b+i(-\sin (j \theta) a+\cos (j \theta) b)) \\
& =\cos (j t / m)(\cos (j \theta) a+\sin (j \theta) b)+\sin (j t / m)(-\sin (j \theta) a+\cos (j \theta) b) \\
& =\cos \left(\frac{j}{m}(t+m \theta)\right) a+\sin \left(\frac{j}{m}(t+m \theta)\right) b \\
& =e^{i \theta}(\cos (j t / m) a+\sin (j t / m) b)=e^{i \theta} \psi_{j}(a+i b)=e^{i \theta} \psi_{j}(\mathbf{z}) .
\end{aligned}
$$

Consider $j$-th irreducible $O(2)$-representation $\mathcal{W}_{j} \simeq \mathbb{C}, j>0$, where for $e^{i \theta} \in S O(2)$, $e^{i \theta} z:=e^{i \theta j} \cdot z$ and $\kappa z:=\bar{z}, z \in \mathbb{C}$. Clearly, since $D_{m} \leq O(2), \mathcal{W}_{j}$ is a $D_{m}$-representation. Put $\mathfrak{s}:=\left\lfloor\frac{m+1}{2}\right\rfloor$. The irreducible $D_{m}$-representations $\mathcal{V}_{i}$ are:

- if $i=0$, then $\mathcal{V}_{0} \simeq \mathbb{R}$ with the trivial $D_{m}$-action;

- if $0<i<m / 2$, then $\mathcal{V}_{i} \simeq \mathbb{R}^{2}=\mathbb{C}$, where $\gamma z=\gamma^{i} \cdot z, \kappa z=\bar{z}, z \in \mathbb{C}$;

- if $i=\mathfrak{s}$, then $\mathcal{V}_{\mathfrak{s}} \simeq \mathbb{R}$ with the $D_{m}$-action $\gamma x=x, \kappa x=-x, x \in \mathbb{R}$;

- if $m$ is even, then we have the irreducible $D_{m}$-representation $\mathcal{V}_{\mathfrak{s}+1} \simeq \mathbb{R}$ with the $D_{m}$ action $\gamma x=-x, \kappa x=x, x \in \mathbb{R}$;

- if $m$ is even, then we have the representation $\mathcal{V}_{\mathfrak{s}+2} \simeq \mathbb{R}$ with $D_{m}$-action $\gamma x=-x$, $\kappa x=-x, x \in \mathbb{R}$.

For the group $D_{m} \times \mathbb{Z}_{2}$, the corresponding irreducible representations (with non-trivial $\mathbb{Z}_{2}$-action) will be denoted by $\mathcal{V}_{i}^{-}$.

Proposition 2.2. The $D_{m}$-representation $\mathcal{W}_{j}$ has the following $D_{m}$-isotypic decomposition

- $\mathcal{W}_{m j} \simeq \mathcal{V}_{0} \oplus \mathcal{V}_{\mathfrak{s}}$ 
- for $0<i<\frac{m}{2}, \mathcal{W}_{m j+i} \simeq \mathcal{W}_{m j-i} \simeq \mathcal{V}_{i}$,

- if $m$ is even, $\mathcal{W}_{m j-\frac{m}{2}} \simeq \mathcal{V}_{\mathfrak{s}+1} \oplus \mathcal{V}_{\mathfrak{s}+2}$.

For $j>0$ and $0 \leq l \leq \mathfrak{r}$, we put

$$
\begin{aligned}
\mathbb{V}_{j m, l}^{+} & =\left\{u \in \mathbb{E}: u(t)=\cos (j t) a, a \in V_{l}\right\}, \\
\mathbb{V}_{j m, l}^{-} & =\left\{u \in \mathbb{E}: u(t)=\sin (j t) b, b \in V_{l}\right\}, \\
\mathbb{V}_{j m-\frac{m}{2}, l}^{+} & =\left\{u \in \mathbb{E}: u(t)=\cos \left(\left(j-\frac{1}{2}\right) t\right) a, a \in V_{l}\right\}, \\
\mathbb{V}_{j m-\frac{m}{2}, l}^{-} & =\left\{u \in \mathbb{E}: u(t)=\sin \left(\left(j-\frac{1}{2}\right) t\right) b, b \in V_{l}\right\} .
\end{aligned}
$$

Therefore, we have the following $\Gamma \times D_{m} \times \mathbb{Z}_{2}$-isotypic decomposition of the space $\mathbb{E}$ :

$$
\mathbb{E}=\bigoplus_{l=0}^{\mathfrak{r}} \bigoplus_{i=0}^{\mathfrak{s}^{*}} \mathcal{E}_{i, l}^{-}, \quad s^{*}:=\left\{\begin{array}{ll}
\mathfrak{s} & \text { if } m \text { is odd } \\
\mathfrak{s}+2 & \text { if } m \text { is even }
\end{array} \quad \mathfrak{s}=\left\lfloor\frac{m+1}{2}\right\rfloor,\right.
$$

where

$$
\mathcal{E}_{0, l}^{-}=\mathbb{V}_{0, l} \oplus \overline{\bigoplus_{j=1}^{\infty} \mathbb{V}_{m j, l}^{+},}, \quad \mathcal{E}_{\mathfrak{s}, l}^{-}=\overline{\bigoplus_{j=1}^{\infty} \mathbb{V}_{m j, l}^{-}},
$$

for $0<i<\frac{m}{2}$

$$
\mathcal{E}_{i, l}^{-}=\overline{\bigoplus_{j=0}^{\infty} \mathbb{V}_{m j+i, l}} \oplus \overline{\bigoplus_{j=1}^{\infty} \mathbb{V}_{m j-i, l}},
$$

and if $m$ is even then

$$
\mathcal{E}_{\mathfrak{s}+1, l}^{-}=\overline{\bigoplus_{j=1}^{\infty} \mathbb{V}_{m j-\frac{m}{2}, l}^{+}}, \quad \mathcal{E}_{\mathfrak{s}+2, l}^{-}=\overline{\bigoplus_{j=1}^{\infty} \mathbb{V}_{m j-\frac{m}{2}, l}^{-}} .
$$

The component $\mathcal{E}_{i, l}^{-}\left(0 \leq i \leq \mathfrak{s}^{*}, 0 \leq l \leq \mathfrak{r}\right)$ is modeled on the irreducible $\Gamma \times D_{m} \times \mathbb{Z}_{2^{-}}$ representation

$$
\mathcal{V}_{i, l}^{-}:=\mathcal{V}_{i}^{-} \otimes \mathcal{U}_{l}
$$

Since the operator $L$ is $O(2) \times \mathbb{Z}_{2}$-equivariant isomorphism, thus $L\left(\mathbb{V}_{j}\right)=\mathbb{V}_{j}$ and $\left.L\right|_{\mathbb{V}_{j}}=$ $-\left(j^{2} / m^{2}+1\right) \operatorname{Idv}_{j}$.

\subsection{Linearization of Equation (11) at 0 :}

We make the following additional assumption

$\left(A_{5}\right) \quad$ There exists a symmetric matrix $A: \mathbb{R}^{k} \rightarrow \mathbb{R}^{k}$ such that

$$
\lim _{x \rightarrow 0} \frac{f(t, x)-A x}{|x|}=0
$$

uniformly with respect to $t \in \mathbb{R}$, and for all integers $j \geq 0$ and $\mu \in \sigma(A)$,

$$
\frac{j^{2}}{m^{2}}+\mu \neq 0
$$

We define the linear operator $\mathscr{A}: \mathbb{E} \rightarrow \mathbb{E}$ (associated with $A: \mathbb{R}^{k} \rightarrow \mathbb{R}^{k}$ ) by

$$
\mathscr{A} u:=u-L^{-1}\left(N_{A}(\mathfrak{j}(u)-\mathfrak{j}(u)), \quad u \in \mathbb{E},\right.
$$

where $N_{A}(\varphi)(t):=A(\varphi(t)), t \in \mathbb{R}, \varphi \in C_{2 \pi m}(\mathbb{R} ; V)$. Under the assumption $\left(A_{5}\right)$ the operator $\mathscr{A}: \mathbb{E} \rightarrow \mathbb{E}$ given by (12) is an isomorphism and $D \mathscr{F}(0)=\mathscr{A}$. 
Lemma 2.3. Assume that $f: \mathbb{R} \times \mathbb{R}^{k} \rightarrow \mathbb{R}^{k}$ satisfies the conditions $\left(A_{1}\right)$ - $\left(A_{5}\right)$. Then there exists $\varepsilon>0$ such that the $G$-map $\mathscr{F}: \mathbb{E} \rightarrow \mathbb{E}$ (given by (7D) and $\mathscr{A}: \mathbb{E} \rightarrow \mathbb{E}$ (given by (12)) are $\Omega_{\varepsilon}$-admissibly $G$-homotopic (here $\Omega_{\varepsilon}:=B_{\varepsilon}(0)$ in $\mathbb{E}$ ).

Proof. Define the linear homotopy $\mathfrak{H}:[0,1] \times \mathbb{E} \rightarrow \mathbb{E}$ as $\mathfrak{H}(\lambda, u):=(1-\lambda) \mathscr{A} u+\lambda \mathscr{F}(u), u \in \mathbb{E}$, and suppose for contradiction that there exists a sequence $\left\{\lambda_{n}, u_{n}\right\}$ such that $u_{n} \neq 0, \lambda_{n} \rightarrow \lambda_{o}$ and $u_{n} \rightarrow 0$ as $n \rightarrow \infty$, then we have

$$
\begin{aligned}
0 & =\mathfrak{H}\left(\lambda_{n}, u_{n}\right)=\left(1-\lambda_{n}\right) \mathscr{A} u_{n}+\lambda_{n} F\left(u_{n}\right) \\
& =\mathscr{A}\left(u_{n}\right)+\lambda_{n}\left(\mathscr{F}\left(u_{n}\right)-\mathscr{A} u_{n}\right) .
\end{aligned}
$$

Put $v_{n}:=\frac{u_{n}}{\left\|u_{n}\right\|}$. Then

$$
0=\mathscr{A} v_{n}+\lambda_{n} \frac{\mathscr{F}\left(u_{n}\right)-\mathscr{A} u_{n}}{\left\|u_{n}\right\|}
$$

Since $\left\|u_{n}\right\| \rightarrow 0$ and $\lambda_{n}$ is bounded, thus

$$
\lim _{n \rightarrow \infty} \frac{\mathscr{F}\left(u_{n}\right)-\mathscr{A} u_{n}}{\left\|u_{n}\right\|}=0,
$$

which implies

$$
0=\lim _{n \rightarrow \infty}\left(\mathscr{A} v_{n}\right) .
$$

On the other hand, since $\mathscr{A}=\mathrm{Id}-\mathscr{K}$, where $\mathscr{K}:=L^{-1}(A \mathfrak{j}-\mathfrak{j})$ is a compact operator, one can assume (by passing to a subsequence) that $\mathscr{K} v_{n} \rightarrow v_{o}$, which implies $v_{n} \rightarrow v_{0}$ and $\left\|v_{o}\right\|=1$, so $v_{o} \in \operatorname{Ker} \mathscr{A}$, but this is a contradiction with $\left(A_{5}\right)$.

\subsection{Nagumo Growth Condition:}

The following condition is often referred to as the Nagumo growth condition:

There exists a constant $M>0$ such that

$$
\forall t \in \mathbb{R} \forall_{x \in \mathbb{R}^{k}}|x| \geq M \Rightarrow f(t, x) \bullet x>0 .
$$

We consider the following parametrized (by $\lambda \in[0,1]$ ) modification of system (1):

$$
\left\{\begin{array}{l}
\ddot{u}(t)=\lambda f(t, u(t))+(1-\lambda) u(t), \quad t \in \mathbb{R}, u(t) \in V, \\
u(t)=u(t+2 \pi m), \dot{u}(t)=\dot{u}(t+2 \pi m) .
\end{array}\right.
$$

Then we have:

Lemma 2.4. Assume that $f: \mathbb{R} \oplus \mathbb{R}^{k} \rightarrow \mathbb{R}^{k}$ is a continuous function satisfying conditions $\left(A_{1}\right)-\left(A_{4}\right)$ and $\left(A_{6}\right)$. If $u(t)$ is a $2 \pi m$-periodic function of class $C^{2}$ such that $\max _{t \in \mathbb{R}}|u(t)| \geq$ $M$ (where $M$ is given in $\left.\left(A_{6}\right)\right)$, then $u(t)$ cannot be a solution of (13) for $\lambda \in[0,1]$.

Proof. Assume for the contradiction that $u(t)$ is a solution while $\max _{t \in \mathbb{R}}|u(t)| \geq M$. Consider the function $\phi(t):=\frac{1}{2}|u(t)|^{2}$. Suppose that $\phi\left(t_{0}\right)=\max _{t \in \mathbb{R}} \phi(t)$, then $\phi^{\prime}\left(t_{0}\right)=u\left(t_{0}\right) \bullet \dot{u}\left(t_{0}\right)=0$ and $\phi^{\prime \prime}\left(t_{0}\right)=\dot{u}\left(t_{0}\right) \bullet \dot{u}\left(t_{0}\right)+\ddot{u}\left(t_{0}\right) \bullet u\left(t_{0}\right) \leq 0$. However, by condition $\left(A_{6}\right), \phi^{\prime \prime}\left(t_{0}\right)=\dot{u}\left(t_{0}\right) \bullet$ $\dot{u}\left(t_{0}\right)+\ddot{u}\left(t_{0}\right) \bullet u\left(t_{0}\right)=\left(\lambda\left(f\left(u\left(t_{0}\right)\right)-u\left(t_{0}\right)\right)+u\left(t_{0}\right)\right) \bullet u\left(t_{0}\right)+\dot{u}\left(t_{0}\right) \bullet \dot{u}\left(t_{0}\right)>(1-\lambda) u\left(t_{0}\right) \bullet u\left(t_{0}\right)+$ $\lambda f\left(u\left(t_{0}\right)\right) \bullet u\left(t_{0}\right)>0$, which leads to a contradiction with condition $\left(A_{6}\right)$.

Lemma 2.5. Assume that $f: \mathbb{R} \oplus \mathbb{R}^{k} \rightarrow \mathbb{R}^{k}$ is a continuous function satisfying conditions $\left(A_{1}\right)-\left(A_{3}\right)$ and $\left(A_{6}\right)$. Then there exists $R>0$ such that for every solution $u \in \mathbb{E}$ to (13), $\lambda \in[0,1]$, we have $\|u\|<R$. In addition, for $\Omega_{R}:=B_{R}(0)$, the map $\mathscr{F}: \mathbb{E} \rightarrow \mathbb{E}$ is $\Omega_{R^{-}}$ admissibly G-homotopic to Id. 
Proof. By Lemma 2.4 there exists a $M>0$ such that any $2 \pi m$-periodic solution $u(t)$ to (13) satisfies $|u(t)|<M$. Take $A_{R}:=\left\{(t, x) \in[0,2 \pi m] \times \mathbb{R}^{k}:|x| \leq M\right\}$. Since the function $F:[0,1] \times \mathbb{R} \oplus \mathbb{R}^{k} \rightarrow \mathbb{R}^{k}$ given by

$$
F(\lambda, t, x)=\lambda f(t, x)+(1-\lambda) x, \quad x \in \mathbb{R}^{k}, \lambda \in[0,1]
$$

is continuous, and the set $[0,1] \times A_{R}$ is compact, then for every solution $u(t)$ to (13) we have

$$
|\ddot{u}(t)|=|F(\lambda, t, u)| \leq \sup \left\{|F(\lambda, t, x)|:(t, x) \in A_{R}, \lambda \in[0,1]\right\}=: M_{2} .
$$

Put $\dot{u}(t)=\left(u_{1}^{\prime}(t), u_{2}^{\prime}(t), \ldots, u_{k}^{\prime}(t)\right)^{T}$. Then for every $1 \leq l \leq k$, since the function $\dot{u}_{l}(t)$ is periodic, there exists $\tau_{o} \in[0,2 \pi m]$ such that $u_{l}^{\prime}\left(\tau_{o}\right)=0$. Thus the identity

$$
u_{l}^{\prime}(t)=\int_{\tau_{o}}^{t} u_{l}^{\prime \prime}(s) d s, \quad t \in \mathbb{R}
$$

implies $\left|u_{l}^{\prime}(t)\right| \leq 2 \pi m M_{2}$ for $t \in \mathbb{R}$ and consequently

$$
\|\dot{u}\|_{\infty}=\max _{t \in \mathbb{R}} \sqrt{\left|u_{1}^{\prime}(t)\right|^{2}+\left|u_{2}^{\prime}(t)\right|^{2}+\cdots+\left|u_{k}^{\prime}(t)\right|^{2}} \leq \sqrt{k} 2 \pi m M_{2}=: M_{1} .
$$

Consequently,

$$
\|\dot{u}\|=\max \left\{\|u\|_{\infty},\|\dot{u}\|_{\infty},\|\ddot{u}\|_{\infty}\right\} \leq \max \left\{M, M_{1}, M_{2}\right\}<\max \left\{M, M_{1}, M_{2}\right\}+1=: R,
$$

and the conclusion follows.

\subsection{Abstract Existence Result}

Assume that $f: \mathbb{R} \oplus V \rightarrow V$ satisfies the assumptions $\left(A_{1}\right)-\left(A_{6}\right)$. We denote the set of negative eigenvalues of the operator $\mathscr{A}$ by $\sigma_{-}(\mathscr{A})$. Then by Lemma 2.3 there exists a sufficiently small $\varepsilon>0$ such that $\mathscr{F}$ is $\Omega_{\varepsilon}$-admissibly $G$-homotopic to $\mathscr{A}$ (given by (12)) and therefore

$$
G-\operatorname{deg}\left(\mathscr{F}, \Omega_{\varepsilon}\right)=G-\operatorname{deg}(\mathscr{A}, B(\mathbb{E}))=\prod_{\lambda \in \sigma_{-}(\mathscr{A})} G-\operatorname{deg}\left(-\left.\operatorname{Id}\right|_{E(\lambda)}, B(E(\lambda))\right),
$$

where $E(\lambda)$ denotes the eigenspace of $\mathscr{A}$ corresponding to $\lambda$, and $B(E(\lambda))$ stands for an open unit ball in $E(\lambda)$.

In order to use the formula (15) we need to compute the negative spectrum $\sigma_{-}(\mathscr{A})$. Since $\mathscr{A}$ is $\Gamma \times O(2) \times \mathbb{Z}_{2}$-equivariant, one can use the isotypic decomposition (9) in order to determine eigenvalues (and eigenspaces) of $\mathscr{A}$ :

$$
\sigma(\mathscr{A})=\left\{\lambda_{j, \mu}:=1+\frac{m^{2}(\mu-1)}{j^{2}+m^{2}}: j=0,1,2, \ldots, \mu \in \sigma(A)\right\} .
$$

Clearly,

$$
\lambda_{j, \mu}=\frac{j^{2}+m^{2} \mu}{j^{2}+m^{2}}<0
$$

if and only if $\mu<-j^{2} / m^{2}$. Notice that, in such a case we also have

$$
\lambda_{0, \mu}<\lambda_{1, \mu}<\cdots<\lambda_{j-1, \mu}<\lambda_{j, \mu}<\cdots<\lambda_{\mathfrak{j}_{\mu}, \mu}<0<\lambda_{\mathfrak{j}_{\mu}+1, \mu},
$$

where $\mathfrak{j}_{\mu}$ is the integer number (by condition $\left(A_{5}\right)$ ) satisfying

$$
-\frac{\left(\mathfrak{j}_{\mu}+1\right)^{2}}{m^{2}}<\mu<-\frac{\mathfrak{j}_{\mu}^{2}}{m^{2}}
$$


On the other hand, by Lemma 2.5. there exists a sufficiently large $R>0$ such that $\mathscr{F}$ is $\Omega_{R}$-admissibly $G$-homotopic to Id. Therefore, $G-\operatorname{deg}\left(\mathscr{F}, \Omega_{R}\right)=G$-deg $\left(\mathrm{Id}, \Omega_{R}\right)=(G)$. Put $\Omega:=\Omega_{R} \backslash \overline{\Omega_{\varepsilon}}$. Then the $G-\operatorname{deg}(\mathscr{F}, \Omega)$ is well defined and by additivity property we have

$$
\begin{aligned}
G-\operatorname{deg}(\mathscr{F}, \Omega) & =G-\operatorname{deg}\left(\mathscr{F}, \Omega_{R}\right)-G-\operatorname{deg}\left(\mathscr{F}, \Omega_{\varepsilon}\right) \\
& =(G)-G-\operatorname{deg}(\mathscr{A}, B(\mathbb{E})) .
\end{aligned}
$$

In this way we can formulate the following abstract existence result:

Theorem 2.6. Assume that $f: \mathbb{R} \oplus V \rightarrow V$ satisfies the assumptions $\left(A_{1}\right)-\left(A_{6}\right), R>0$ is a sufficiently large (given by Lemma 2.5), $\varepsilon>0$ is sufficiently small (given by Lemma 2.3) and $\Omega:=\Omega_{R} \backslash \overline{\Omega_{\varepsilon}}$. If the G-equivariant degree

$$
G-\operatorname{deg}(\mathscr{F}, \Omega)=n_{1}\left(H_{1}\right)+n_{2}\left(H_{2}\right)+\cdots+n_{s}\left(H_{s}\right) \in A(G)
$$

has a non-zero coefficient $n_{j}$, then there exists a $2 \pi m$-periodic solution $u \in \Omega$ to (1) such that $G_{u} \leq H_{j}$. In addition, if $D_{m} \not \leq H_{j}$ then $u$ is non-constant, and if for some $g \in D_{m}, g \neq 1$, we have $(g,-1) \in H_{j}$, then the solution $u$ can not be $2 \pi$-periodic solution, i.e. its minimal period is not $2 \pi$.

Proof. The existence of a $2 \pi m$-periodic solution $x$ is a direct consequence of the existence property for $G$-equivariant degree. Moreover, if $u(t)$ is constant, then clearly $u(t+l 2 \pi)=u(t)$ and $u(-t)=u(t)$, for $t \in \mathbb{R}$ and $l \in \mathbb{Z}$, so $D_{m} \leq H_{j}$. Assume that there exists an element $(g,-1) \in H_{j}$ for some $1 \neq g \in D_{m}$, which implies that for some $1 \leq l \leq m-1$ we have $g=\gamma^{l}$ or $g=\gamma^{l} \kappa$. Then we also have

$$
\forall_{t \in \mathbb{R}}((g,-1) u)(t)=u(t) \Rightarrow x(0)=-x(2 \pi l) .
$$

Since $x \not \equiv 0$ it follows that $u(t) \neq u(t+l 2 \pi)$ and consequently $u(t) \neq u(t+2 \pi)$.

\subsection{Subharmonic Solutions in Non-Equivariant Case}

To illustrate our previous theorem, in this section we assume that $\Gamma=\{e\}$, i.e. we consider the case of (1) without additional symmetries and $G=D_{m} \times \mathbb{Z}_{2}$.

Definition 2.7. We define

$$
i(j):= \begin{cases}\alpha(j) & \text { if } \alpha(j) \leq\left\lfloor\frac{m}{2}\right\rfloor, \\ m-\alpha(j) & \text { if } \alpha(j)>\left\lfloor\frac{m}{2}\right\rfloor,\end{cases}
$$

where $\alpha(j) \in\{0,1, \ldots, m-1\}$ satisfies $\alpha(j) \equiv j(\bmod m)$, i.e.

$$
\alpha(j):=j-\left\lfloor\frac{j}{m}\right\rfloor m \in\{0,1,2, \ldots, m-1\} .
$$

We use notation $\mathfrak{m}(\mu)$ for the algebraic multiplicity of $\mu$ belonging to the spectrum of $A$. The negative spectrum $\sigma_{-}(\mathscr{A})$ can be represented as

$$
\sigma_{-}(\mathscr{A})=\bigcup_{\mu \in \sigma_{-}(A)}\left\{\lambda_{0, \mu}, \lambda_{1, \mu}, \ldots, \lambda_{\mathfrak{j}_{\mu}-1, \mu}, \lambda_{\mathfrak{j}_{\mu}, \mu}\right\}
$$

Denote by $E\left(\lambda_{j, \mu}\right)$ the eigenspace of $\lambda_{j, \mu}$. In order to compute the degree, we introduce the following notation:

$$
\beta_{0}(\mu):=\left(\left\lfloor\frac{\mathfrak{j}_{\mu}}{m}\right\rfloor+1\right) \mathfrak{m}(\mu), \quad \beta_{\mathfrak{s}}(\mu):=\left\lfloor\frac{\mathfrak{j}_{\mu}}{m}\right\rfloor \mathfrak{m}(\mu),
$$


and for $i=\mathfrak{s}+1, \mathfrak{s}+2$ (in the case $m$ is even),

$$
\beta_{i}(\mu):= \begin{cases}\left\lfloor\frac{\mathfrak{j}_{\mu}}{m}\right\rfloor \mathfrak{m}(\mu), & \text { if } \alpha\left(\mathfrak{j}_{\mu}\right)<\frac{m}{2}, \\ \left(\left\lfloor\frac{\mathfrak{j}_{\mu}}{m}\right\rfloor+1\right) \mathfrak{m}(\mu), & \text { if } \alpha\left(\mathfrak{j}_{\mu}\right) \geq \frac{m}{2},\end{cases}
$$

and finally for $0<i<\frac{m}{2}$,

$$
\beta_{i}(\mu):= \begin{cases}2\left\lfloor\frac{\mathfrak{j}_{\mu}}{m}\right\rfloor \mathfrak{m}(\mu) & \text { if } \alpha\left(\mathfrak{j}_{\mu}\right)<i, \\ \left.\left(2 \mid \frac{\mathfrak{j}_{\mu}}{m}\right\rfloor+1\right) \mathfrak{m}(\mu) & \text { if } i \leq \alpha\left(\mathfrak{j}_{\mu}\right)<m-i, \\ 2\left(\left\lfloor\frac{\mathfrak{j}_{\mu}}{m}\right\rfloor+1\right) \mathfrak{m}(\mu) & \text { if } m-i \leq \alpha\left(\mathfrak{j}_{\mu}\right) .\end{cases}
$$

Definition 2.8. We define

$$
\eta_{i}:=\sum_{\mu \in \sigma_{-}(A)} \beta_{i}(\mu)
$$

for $i=0,1, \ldots, \mathfrak{s}, \mathfrak{s}+1, \mathfrak{s}+2$. The number $\eta_{i}$ counts the "total number of times" that the irreducible representation $\mathcal{V}_{i}^{-}$appears in $\sigma_{-}(\mathscr{A})$.

We have the following list of basic degrees for the irreducible $G$-representations (see appendix):

- for $0 \leq i \leq\left\lfloor\frac{m}{2}\right\rfloor, h:=\operatorname{gcd}(m, i), m / h$ is odd then

$$
\operatorname{deg}_{\mathcal{V}_{i}^{-}}=\left(D_{m} \times \mathbb{Z}_{2}\right)-\left(D_{h}\right)-\left(D_{h}^{z}\right)+\left(\mathbb{Z}_{h}\right) ;
$$

- if $m / h \equiv 2(\bmod 4)$ then

$$
\operatorname{deg}_{\mathcal{V}_{i}^{-}}=\left(D_{m} \times \mathbb{Z}_{2}\right)-\left(D_{2 h}^{d}\right)-\left(D_{2 h}^{\hat{d}}\right)+\left(\mathbb{Z}_{2 h}^{d}\right) ;
$$

- if $m / h \equiv 0(\bmod 4)$ then

$$
\operatorname{deg}_{\mathcal{V}_{i}^{-}}=\left(D_{m} \times \mathbb{Z}_{2}\right)-\left(D_{2 h}^{d}\right)-\left(\widetilde{D}_{2 h}^{d}\right)+\left(\mathbb{Z}_{2 h}^{d}\right) ;
$$

- if $i=\mathfrak{s}$ then

$$
\operatorname{deg}_{\mathcal{V}_{\mathfrak{s}}^{-}}=\left(D_{m} \times \mathbb{Z}_{2}\right)-\left(D_{m}^{z}\right)
$$

- if $i=0$ then

$$
\operatorname{deg}_{\mathcal{V}_{0}^{-}}=\left(D_{m} \times \mathbb{Z}_{2}\right)-\left(D_{m}\right)
$$

- if $m$ is even and $i=\mathfrak{s}+1$ then

$$
\operatorname{deg}_{\mathcal{V}_{\mathfrak{s}+1}^{-}}=\left(D_{m} \times \mathbb{Z}_{2}\right)-\left(D_{m}^{d}\right)
$$

- if $m$ is even and $i=\mathfrak{s}+2$ then

$$
\operatorname{deg}_{\mathcal{V}_{\mathfrak{s}+2}^{-}}=\left(D_{m} \times \mathbb{Z}_{2}\right)-\left(D_{m}^{\hat{d}}\right)
$$

Notice that $\operatorname{deg}_{\mathcal{V}_{i}^{-}}=\operatorname{deg}_{\mathcal{V}_{i^{\prime}}^{-}}$if and only if $\operatorname{gcd}(i, m)=\operatorname{gcd}\left(i^{\prime}, m\right)$. Therefore, we introduce the numbers $\rho_{i}, 0 \leq i \leq \mathfrak{s}+2$, that will allow us to determine how many times the basic degree $\operatorname{deg}_{\mathcal{V}_{i}^{-}}$appears in the degree of $G-\operatorname{deg}(\mathscr{A}, B(\mathbb{E}))$.

Definition 2.9. We define $\rho_{0}:=\eta_{0}, \rho_{\mathfrak{s}}:=\eta_{\mathfrak{s}}, \rho_{\mathfrak{s}+1}:=\eta_{\mathfrak{s}+1}, \rho_{\mathfrak{s}+2}:=\eta_{\mathfrak{s}+2}$, and

$$
\rho_{i}:=\sum_{\operatorname{gcd}\left(i^{\prime}, m\right)=\operatorname{gcd}(i, m)} \eta_{i^{\prime}}, \quad 0<i<\frac{m}{2} .
$$


Before proving our main theorem, we need to analyze the maximal $G$-orbit types in the space $\mathbb{E} \backslash\{0\}$.

Lemma 2.10. Suppose $m=2^{\mathfrak{n}} m^{\prime}$, where $m^{\prime}$ is an odd integer. Then the maximal orbit types in $\mathbb{E} \backslash\{0\}$ are:

(a) $\left(D_{m}^{z}\right),\left(D_{m}\right)$, and if $\mathfrak{n}>0$,

(b) $\left(D_{m}^{d}\right),\left(\widetilde{D}_{m}^{d}\right),\left(D_{\frac{m}{2}}^{d}\right),\left(\widetilde{D}_{\frac{m}{2}}^{d}\right), \ldots,\left(D_{\frac{m}{2^{\mathfrak{n}-1}}}^{d}\right),\left(\widetilde{D}_{\frac{m}{2^{\mathfrak{n}}-1}}^{d}\right)$,

Proof. The maximal $G$-orbit types in $\mathbb{E} \backslash\{0\}$ are exactly the same as the maximal $G$-orbit types which occur in the space $\mathcal{V}^{*} \backslash\{0\}$, where

$$
\mathcal{V}^{*}:=\mathcal{V}_{0}^{-} \oplus \mathcal{V}_{1}^{-} \oplus \mathcal{V}_{2}^{-} \oplus \mathcal{V}_{\mathfrak{s}}^{-} \oplus \mathcal{V}_{\mathfrak{s}+1}^{-} \oplus \mathcal{V}_{\mathfrak{s}+2}^{-}
$$

First we identify the maximal orbit types in $\mathcal{V}_{i}^{-} \backslash\{0\}, i=0,1, \ldots, \mathfrak{s}+2$,

- for $0 \leq i \leq\left\lfloor\frac{m}{2}\right\rfloor, h:=\operatorname{gcd}(m, i), p_{h}:=\frac{m}{h}$ and if $p_{h}$ is odd, then the orbit types are: $\left(D_{h}\right),\left(D_{h}^{z}\right)$;

- if $p_{h} \equiv 2(\bmod 4)$, then the maximal orbit types are: $\left(\left(D_{2 h}^{d}\right),\left(D_{2 h}^{\hat{d}}\right)\right.$;

- if $p_{h} \equiv 0(\bmod 4)$, then the maximal orbit types are: $\left(D_{2 h}^{d}\right),\left(\widetilde{D}_{2 h}^{d}\right)$;

- if $i=\mathfrak{s}$, then the maximal orbit type is: $\left(D_{m}^{z}\right)$;

- if $i=0$, then the maximal orbit type is: $\left(D_{m}\right)$;

- if $m$ is even and $i=\mathfrak{s}+1$, then the maximal orbit type is: $\left(D_{m}^{d}\right)$;

- if $m$ is even and $i=\mathfrak{s}+2$, then the maximal orbit type is: $\left(D_{m}^{\hat{d}}\right)$.

Notice that $\left(D_{m}\right)$ and $\left(D_{m}^{z}\right)$ are the maximal orbit types which occur in $\mathcal{V}_{0}^{-} \oplus \mathcal{V}_{\mathfrak{s}}^{-} \backslash\{0\}$. On the other hand for $m$ being an even integer, we have (see Table 5.3 in [2]):

- $\left(D_{2 n}^{d} \leq\left(D_{m}^{d}\right)\right.$ if and only if $n \mid \frac{m}{2}$ and $\frac{m}{2 n}$ is odd;

- $\left(\widetilde{D}_{2 n}^{d} \leq\left(\widetilde{D}_{m}^{d}\right)\right.$ if and only if $n \mid \frac{m}{2}$ and $\frac{m}{2 n}$ is odd;

- $\left(D_{2 n}^{\hat{d}} \leq\left(D_{m}^{\hat{d}}\right)\right.$ if and only if $n \mid \frac{m}{2}$ and $\frac{m}{4 n}$ is odd;

and the maximality of the orbit types $\left(D_{m}^{d}\right),\left(\widetilde{D}_{m}^{d}\right),\left(D_{\frac{m}{2}}^{d}\right),\left(\widetilde{D}_{\frac{m}{2}}^{d}\right), \ldots,\left(D_{\frac{m}{2^{\mathfrak{n}}-1}}^{d}\right),\left(\widetilde{D}_{\frac{m}{2^{\mathfrak{n}-1}}}^{d}\right)$ follows from this result.

We have the main theorem.

Theorem 2.11. Let $m$ be a natural number and $f: \mathbb{R} \times \mathbb{R}^{k} \rightarrow \mathbb{R}^{k}$ be a continuous function satisfying the assumptions $\left(A_{1}\right)-\left(A_{3}\right)$ and $\left(A_{5}\right)-\left(A_{6}\right)$. Suppose $m=2^{\varepsilon_{0}} p_{1}^{\varepsilon_{1}} p_{2}^{\varepsilon_{2}} \ldots p_{s}^{\varepsilon_{s}}$, where $\varepsilon_{0} \geq 0, \varepsilon_{l}>0$, and $p_{l}, l=1,2, \ldots, s$, are the prime numbers such that $2<p_{1}<p_{2}<\cdots<p_{s}$. For $l=1,2, \ldots s$ put $m_{l}:=\frac{m}{p_{l}}$. Then

(i) if $\rho_{0}$ is odd, then the system (1) admits a G-orbit of $2 \pi m$-periodic solutions with symmetries $\left(D_{m}\right)$, and

(ii) if $\rho_{\mathfrak{s}}$ is odd, then the system (11) admits a G-orbit of $2 \pi m$-periodic solutions with symmetries $\left(D_{m}^{z}\right)$, and

(iii) if for some $l=1,2, \ldots, s, \rho_{m_{l}}$ is odd, then the system (1) admits a $G$-orbit of $2 \pi m$ periodic solutions with symmetries either $\left(D_{m_{i}}^{z}\right)$ or $\left(D_{m}^{z}\right)$, and

(iv) if $\varepsilon_{0}>0$, and $\rho_{\mathfrak{s}+1}$ is odd, then the system (1) admits a $G$-orbit of $2 \pi m$-periodic solutions with symmetries exactly $\left(D_{m}^{d}\right)$, and

(iv) if $\varepsilon_{0}>0$, and $\rho_{\mathfrak{s}+2}$ is odd, then the system (11) admits a G-orbit of $2 \pi m$-periodic solutions with symmetries exactly $\left(D_{m}^{\hat{d}}\right)$, and 
(iv) if $\varepsilon_{0}=\mathfrak{k}>0$, and for some $2 \leq k \leq \mathfrak{k}, \rho \frac{m}{2^{k}}$ is odd, then the system (1) admits a $G$-orbit of $2 \pi m$-periodic solutions with symmetries exactly $\left(D_{\frac{m}{2^{k-1}}}^{d}\right)$ and $\left(D_{\frac{m}{2^{k-1}}}^{\hat{d}}\right)$.

Proof. Clearly for $\mu \in \sigma_{-}(A)$ we have the following formula for the $\mathcal{V}_{i}^{-}$-isotypic multiplicity of the eigenvalue $\lambda_{j, \mu}$

$$
m_{i}^{-}\left(\lambda_{j, \mu}\right)= \begin{cases}\mathfrak{m}(\mu) & \text { if } i=i(j), 0<i<\frac{m}{2} \\ \mathfrak{m}(\mu) & \text { if } i=\mathfrak{s}, j>0, i(j)=0 \\ \mathfrak{m}(\mu) & \text { if } i=\mathfrak{s}+1, i(j)=\frac{m}{2} \\ \mathfrak{m}(\mu) & \text { if } i=\mathfrak{s}+2, i(j)=\frac{m}{2} \\ 0 & \text { otherwise. }\end{cases}
$$

Therefore (by (20)), we have

$$
G-\operatorname{deg}(\mathscr{A}, B(\mathbb{E}))=\prod_{\mu \in \sigma_{-}(A)} \prod_{j=0}^{\mathrm{j}_{\mu}} G-\operatorname{deg}\left(-\mathrm{Id}, B\left(E\left(\lambda_{j, \mu}\right)\right),\right.
$$

where

$$
G-\operatorname{deg}\left(-\operatorname{Id}, B\left(E\left(\lambda_{j, \mu}\right)\right)= \begin{cases}\left(\operatorname{deg}_{\mathcal{V}_{0}^{-}}\right)^{\mathfrak{m}(\mu)} & \text { if } j=0, \\ \left(\operatorname{deg}_{\mathcal{V}_{0}^{-}}\right)^{\mathfrak{m}(\mu)} \circ\left(\operatorname{deg}_{\mathcal{V}_{\mathfrak{s}}^{-}}\right)^{\mathfrak{m}(\mu)} & \text { if } j>0, i(j)=0, \\ \left(\operatorname{deg}_{\mathcal{V}_{i}^{-}}\right)^{\mathfrak{m}(\mu)} & \text { if } 0<i=i(j)<\frac{m}{2}, \\ \left(\operatorname{deg}_{\mathcal{V}_{\mathfrak{s}+1}^{-}}\right)^{\mathfrak{m}(\mu)} \circ\left(\operatorname{deg}_{\mathcal{V}_{\mathfrak{s}+2}^{-}}\right)^{\mathfrak{m}(\mu)} & \text { if } i(j)=\frac{m}{2} .\end{cases}\right.
$$

Consequently, we obtain the following formula:

$$
\begin{aligned}
G-\operatorname{deg}(\mathscr{F}, \Omega) & =(G)-G-\operatorname{deg}(\mathscr{A}, B(\mathbb{E})) \\
& =(G)-\prod_{\mu \in \sigma_{-}(A)} \prod_{i=0}^{\mathfrak{s}+2}\left(\operatorname{deg}_{\mathcal{V}_{i}^{-}}\right)^{\beta_{i}(\mu)} .
\end{aligned}
$$

Since $\eta_{i}$ counts the "total number of times" that the irreducible representation $\mathcal{V}_{i}^{-}$appears in $\sigma_{-}(\mathscr{A})$, we obtain that

$$
G-\operatorname{deg}(\mathscr{F}, \Omega)=(G)-\prod_{i=0}^{\mathfrak{s}+2}\left(\operatorname{deg}_{\mathcal{V}_{i}^{-}}\right)^{\eta_{i}}
$$

Since

$$
\operatorname{deg}_{\mathcal{V}_{i}^{-}}=\operatorname{deg}_{\mathcal{V}_{i^{\prime}}^{-}} \quad \Leftrightarrow \quad \operatorname{gcd}(i, m)=\operatorname{gcd}\left(i^{\prime}, m\right),
$$

and the numbers $\rho_{i}$ indicate the number of occurrences of the basic degree $\operatorname{deg}_{\mathcal{V}_{i}^{-}}$in the product (23), the conclusion follows from Lemma 2.10 and the fact that the square of any basic degree is the unit element $(G) \in A(G)$, i.e. $\left(\operatorname{deg}_{\mathcal{V}_{i}^{-}}\right)^{2}=(G)$.

In order to illustrate the applications of Theorem 2.12 we only consider a simple case when the operator $A$ has one simple negative eigenvalue $\mu$ satisfying

$$
-\frac{(\mathfrak{p}+1)^{2}}{m^{2}}<\mu<-\frac{\mathfrak{p}^{2}}{m^{2}}, \quad \mathfrak{p}:=\left\lfloor\frac{m}{2}\right\rfloor .
$$

Then we get the following result:

Corollary 2.12. Let $m$ be a natural number and $f: \mathbb{R} \times \mathbb{R}^{k} \rightarrow \mathbb{R}^{k}$ be a continuous function satisfying the assumptions $\left(A_{1}\right)-\left(A_{3}\right),\left(A_{5}\right)-\left(A_{6}\right)$ and $\sigma_{-}(A)$ consists a single simple eigenvalue $\mu$ satisfying (24). Suppose $m=2^{\varepsilon_{0}} p_{1}^{\varepsilon_{1}} p_{2}^{\varepsilon_{2}} \ldots p_{s}^{\varepsilon_{s}}$, where $p_{l}>2$ are distinct prime numbers, $\varepsilon_{0} \geq 0, \varepsilon_{l} \geq 1$ for $l=1,2, \ldots, s$. Then 
(i) the system (1) admits $G$-orbit of non-zero $2 \pi m$-periodic solutions with symmetries $\left(D_{m}\right)$;

(ii) if for some $l=1,2, \ldots, s$, the number $\frac{p_{l}-1}{2}$ is odd, the system (11) admits $G$-orbit of $2 \pi m$-periodic solutions with symmetries $\left(D_{m_{l}}^{z}\right)$ or $\left(D_{m}^{z}\right)$ (here $\left.m_{l}=\frac{m}{p_{l}}\right)$;

(iii) if $\varepsilon_{0}>0$, the system (1) admits $G$-orbit of $2 \pi m$-periodic solutions with symmetries exactly $\left(D_{m}^{d}\right),\left(D_{m}^{\hat{d}}\right)$;

(iv) if $\varepsilon_{0}>1$, the system (1) admits $G$-orbit of $2 \pi m$-periodic solutions with symmetries $\operatorname{exactly}\left(D_{\frac{m}{2}}^{d}\right),\left(\widetilde{D}_{\frac{m}{2}}^{d}\right)$;

Proof. Notice that we have

$$
\beta_{0}(\mu)=1, \beta_{\mathfrak{s}}(\mu)=0, \beta_{i}(\mu)=1 \text { for } 0<i<\frac{m}{2},
$$

and in the case $m$ is even

$$
\beta_{\mathfrak{s}}(\mu+1)=\beta_{\mathfrak{s}}(\mu+2)=1 \text {. }
$$

Consequently,

- Since $\rho_{0}=\beta_{0}=1$, thus there exist a non-zero $2 \pi m$-periodic solution to the system (1) with symmetries exactly $\left(D_{m}\right)$;

- Since $\rho_{\mathfrak{s}}=\beta_{\mathfrak{s}}=0$, we cannot conclude the existence of a $2 \pi m$-periodic solution to the system (11) with symmetries exactly $\left(D_{m}^{z}\right)$;

- Since $\rho_{m_{l}}=\beta_{m_{l}} \cdot \frac{p_{l}-1}{2}=\frac{p_{l}-1}{2}, l=1,2, \ldots, s$, if $\frac{p_{l}-1}{2}$ is odd there exists a $2 \pi m$-periodic solution to the system (1) with symmetries either $\left(D_{m_{l}}^{z}\right)$ or $\left(D_{m}^{z}\right)$ (notice that $\left(D_{m_{l}}^{z}\right)$ is not a maximal orbit type);

- In the case $m$ is even, i.e. $\varepsilon_{0}>0$, since $\rho_{\mathfrak{s}+1}=\rho_{\mathfrak{s}+2}=1$, it follows that the system (11) has an orbit of $2 \pi m$-periodic solutions with symmetries either $\left(D_{m}^{d}\right)$ or $\left(D_{m}^{\hat{d}}\right)$;

- In addition, if $\varepsilon_{0}>1$, notice that $\rho \frac{m}{4}=\beta \frac{m}{4}=1$, thus the system (11) admits $G$-orbit of $2 \pi m$-periodic solutions with symmetries $\left(D_{\frac{m}{2}}^{d}\right),\left(\widetilde{D}_{\frac{m}{2}}^{d}\right)$;

In order to illustrate that the other maximal orbit types in in $\mathbb{E} \backslash\{0\}$ can also appear as symmetries of $2 \pi m$-periodic subharmonic solutions to (1), we assume that $m=2^{n} p_{1}^{\varepsilon_{1}} p_{2}^{\varepsilon_{2}} \ldots p_{s}^{\varepsilon_{s}}$, $n>1$, and the operator $A$ has one simple negative eigenvalue $\mu$ satisfying

$$
-\frac{(\mathfrak{p}+1)^{2}}{m^{2}}<\mu<-\frac{\mathfrak{p}^{2}}{m^{2}}, \quad \mathfrak{p}:=\left\lfloor\frac{m}{2^{n}}\right\rfloor .
$$

Then we have:

Corollary 2.13. Let $m$ be a natural number and $f: \mathbb{R} \times \mathbb{R}^{k} \rightarrow \mathbb{R}^{k}$ be a continuous function satisfying the assumptions $\left(A_{1}\right)-\left(A_{3}\right),\left(A_{5}\right)-\left(A_{6}\right)$ and $\sigma_{-}(A)$ consists a single simple eigenvalue $\mu$ satisfying (25). Suppose $m=2^{n} p_{1}^{\varepsilon_{1}} p_{2}^{\varepsilon_{2}} \ldots p_{s}^{\varepsilon_{s}}$, where $n>2, p_{l}>2$ are distinct prime numbers, $\varepsilon_{0} \geq 0, \varepsilon_{l} \geq 1$ for $l=1,2, \ldots, s$. Then

(i) The system (1) admits $G$-orbit of non-zero $2 \pi m$-periodic solutions with symmetries $\left(D_{m}\right)$,

(ii) The system (1) admits $G$-orbit of $2 \pi m$-periodic solutions with symmetries exactly $\left(D_{\frac{m}{2^{n-1}}}^{2^{2}}\right),\left(\widetilde{D}_{\frac{m}{2^{n-1}}}^{d}\right)$; 


\subsection{Examples of Symmetric Systems}

In this section we assume that $k=3, f: \mathbb{R} \times \mathbb{R}^{3} \rightarrow \mathbb{R}^{3}$ satisfies assumptions $\left(A_{1}\right)-\left(A_{5}\right)$ with $\Gamma=D_{3}$ and

$$
A=\frac{1}{4}\left[\begin{array}{lll}
-4 & -2 & -2 \\
-2 & -4 & -2 \\
-2 & -2 & -4
\end{array}\right]
$$

Then we have

$$
\sigma(A)=\left\{\mu_{0}=-2, \mu_{1}=-\frac{1}{2}\right\} \text {. }
$$

Case $m=3: \quad$ In this case $G=D_{3} \times D_{3} \times \mathbb{Z}_{2}$ and we have

$$
\sigma(\mathscr{A})=\left\{\lambda_{j, l}:=1+\frac{9\left(\mu_{l}-1\right)}{j^{2}+9}: l=0,1, j=0,1,2, \ldots\right\}
$$

Then

$$
\begin{gathered}
\sigma_{-}(\mathscr{A})=\left\{\lambda_{0,0}=-2, \lambda_{1,0}=-\frac{17}{10}, \lambda_{2,0}=-\frac{14}{13}, \lambda_{3,0}=-\frac{1}{2}, \lambda_{4,0}=-\frac{2}{25},\right. \\
\left.\lambda_{0,1}=-\frac{1}{2}, \lambda_{1,1}=-\frac{7}{20}, \lambda_{2,1}=-\frac{1}{26}\right\},
\end{gathered}
$$

and each of the eigenvalues $\lambda_{l, i}$ are isotypicaly simple, i.e. the eigenspaces $E\left(\lambda_{l, i}\right)$ are irreducible $G$-representations. More precisely, we have

$$
\begin{array}{cl}
E\left(\lambda_{0,0}\right) \simeq \mathcal{V}_{0,0}^{-}, \quad & E\left(\lambda_{3,0}\right) \simeq \mathcal{V}_{0,0}^{-} \oplus \mathcal{V}_{2,0}^{-}, \quad E\left(\lambda_{1,0}\right) \simeq E\left(\lambda_{2,0}\right) \simeq E\left(\lambda_{4,0}\right) \simeq \mathcal{V}_{1,0}^{-} \\
E\left(\lambda_{0,1}\right) \simeq \mathcal{V}_{1,0}^{-}, \quad E\left(\lambda_{1,1}\right) \simeq E\left(\lambda_{2,1}\right) \simeq \mathcal{V}_{1,1}^{-} .
\end{array}
$$

Therefore, we obtain

$$
\begin{aligned}
G-\operatorname{deg}(\mathscr{F}, \Omega) & =(G)-G-\operatorname{deg}\left(\mathscr{A}, B_{1}(0)\right) \\
& =(G)-\left(\operatorname{deg}_{\mathcal{V}_{0,0}^{-}}\right)^{2} \circ \operatorname{deg}_{\mathcal{V}_{2,0}^{-}} \circ\left(\operatorname{deg}_{\mathcal{V}_{1,0}^{-}}\right)^{3} \circ \operatorname{deg}_{\mathcal{V}_{0,1}^{-}} \circ\left(\operatorname{deg}_{\mathcal{V}_{1,1}^{-}}\right)^{2} \\
& =(G)-\operatorname{deg}_{\mathcal{V}_{0,1}^{-}} \circ \operatorname{deg}_{\mathcal{V}_{1,0}^{-}} \circ \operatorname{deg}_{\mathcal{V}_{2,0}^{-}}
\end{aligned}
$$

GAP Code: We use GAP package EquiDeg to compute $G-\operatorname{deg}(\mathscr{F}, \Omega)$ for the groups $G:=$ $D_{3} \times D_{3} \times \mathbb{Z}_{2}$. The GAP code $\mathrm{f}$ is given below.

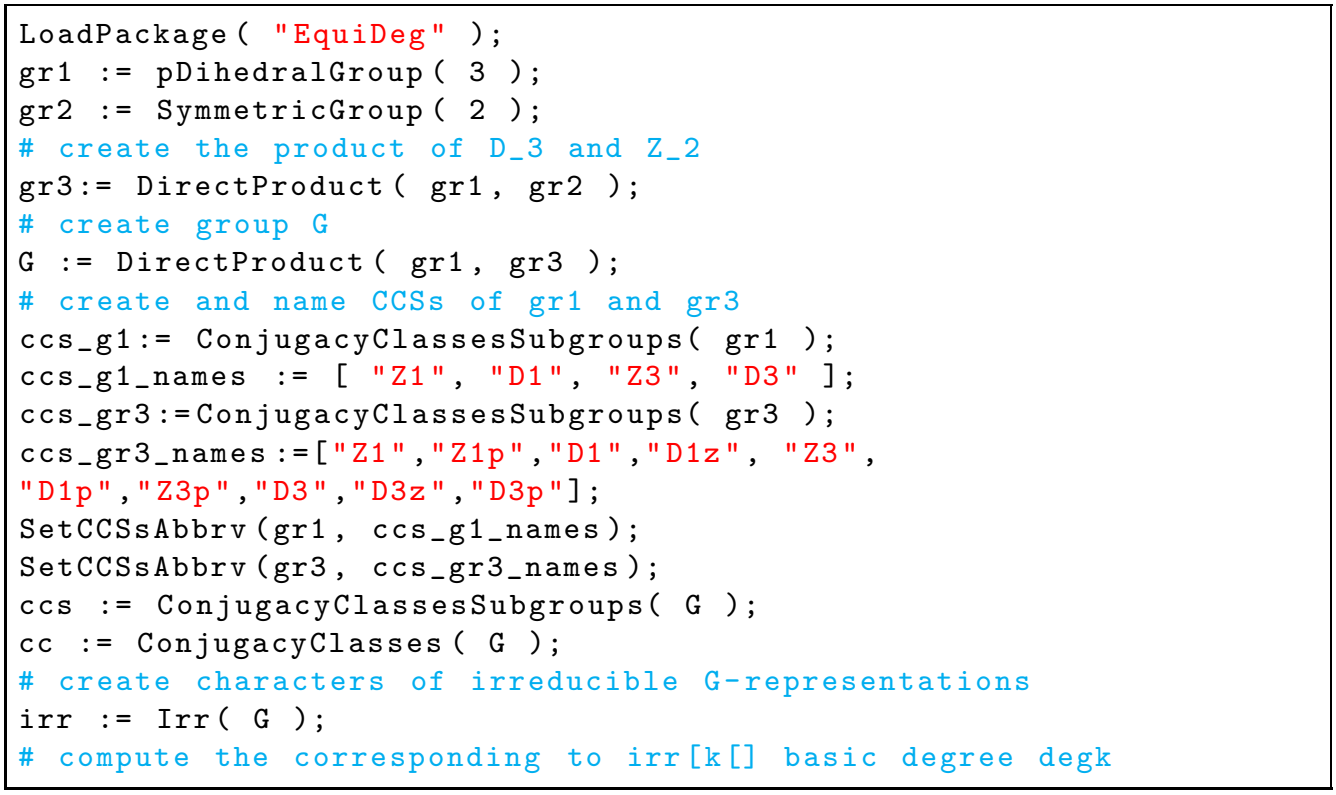


For a subgroup $K \leq D_{n}$, we use the symbol $K^{p}:=K \times \mathbb{Z}_{2}$, but in the code we simply write Kp. By using the list of conjugacy classes cc, one can easily recognize the irreducible $G$-representations $\mathcal{V}_{i, l}^{ \pm}:=\mathcal{V}_{i}^{ \pm} \otimes \mathcal{U}_{l}$. For example, the character

\begin{tabular}{|l|ccccccccc|}
\hline & $( \pm \mathbf{1})$ & $\left( \pm \kappa_{2}\right)$ & $\left( \pm \boldsymbol{\gamma}_{2}\right)$ & $\left( \pm \kappa_{1}\right)$ & $\left( \pm \boldsymbol{\kappa}_{1} \kappa_{2}\right)$ & $\left( \pm \kappa_{1} \gamma_{2}\right)$ & $\left( \pm \gamma_{1}\right)$ & $\left( \pm \gamma_{1} \kappa_{2}\right)$ & $\left( \pm \gamma_{1} \gamma_{2}\right)$ \\
\hline $\operatorname{Irr}(\mathrm{G})[9]$ & \pm 2 & $\mp 2$ & \pm 2 & 0 & 0 & 0 & $\mp 1$ & \pm 1 & $\mp 1$ \\
\hline
\end{tabular}

is the character of the $G$-irreducible representation $\mathcal{V}_{2,1}^{-}$. To be more precise, we have the following correspondence of the characters.

\begin{tabular}{|l|l|l|}
\hline $\operatorname{Irr}(G)[1]=\chi_{\mathcal{V}_{0,0}^{+}}$ & $\operatorname{Irr}(G)[7]=\chi_{\mathcal{V}_{2,0}^{+}}$ & $\operatorname{Irr}(G)[13]=\chi_{\mathcal{V}_{1,2}^{-}}$ \\
$\operatorname{Irr}(G)[2]=\chi_{\mathcal{V}_{2,2}^{-}}$ & $\operatorname{Irr}(G)[8]=\chi_{\mathcal{V}_{0,2}^{+}}$ & $\operatorname{Irr}(G)[14]=\chi_{\mathcal{V}_{1,0}^{-}}$ \\
$\operatorname{Irr}(G)[3]=\chi_{\mathcal{V}_{2,0}^{-}}$ & $\operatorname{Irr}(G)[9]=\chi_{\mathcal{V}_{2,1}^{-}}$ & $\operatorname{Irr}(G)[15]=\chi_{\mathcal{V}_{1,2}^{+}}$ \\
$\operatorname{Irr}(G)[4]=\chi_{\mathcal{V}_{0,2}^{-}}$ & $\operatorname{Irr}(G)[10]=\chi_{\mathcal{V}_{0,1}^{-}}$ & $\operatorname{Irr}(G)[16]=\chi_{\mathcal{V}_{1,0}^{+}}$ \\
$\operatorname{Irr}(G)[5]=\chi_{\mathcal{V}_{0,0}^{-}}$ & $\operatorname{Irr}(G)[11]=\chi_{\mathcal{V}_{2,1}^{+}}$ & $\operatorname{Irr}(G)[17]=\chi_{\mathcal{V}_{1,1}^{+}}$ \\
$\operatorname{Irr}(G)[6]=\chi_{\mathcal{V}_{2,2}^{+}}$ & $\operatorname{Irr}(G)[12]=\chi_{\mathcal{V}_{0,1}^{+}}$ & $\operatorname{Irr}(G)[18]=\chi_{\mathcal{V}_{1,1}^{-}}$ \\
\hline
\end{tabular}

To conclude the computations in GAP (we continue to use the package EquiDeg).

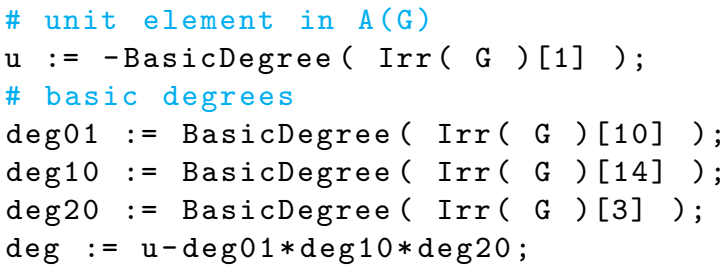

The list of conjugacy classes of $G=D_{3} \times D_{3} \times \mathbb{Z}_{2}$ generated by GAP is $\left\{\left(H_{k}\right): 1 \leq k \leq 69\right\}$, where $(G)=\left(H_{69}\right)$. As a result we obtain

$$
\begin{aligned}
G-\operatorname{deg}(\mathscr{F}, \Omega) & =-\left(H_{1}\right)+\left(H_{4}\right)+\left(H_{6}\right)+\left(H_{8}\right)+\left(H_{11}\right)-2\left(H_{18}\right)-\left(H_{22}\right)-\left(H_{27}\right) \\
& +\left(H_{35}\right)+\left(H_{43}\right)+\left(H_{44}\right)+\left(H_{45}\right)-\left(H_{51}\right)+\left(H_{63}\right)-\left(H_{67}\right),
\end{aligned}
$$

where the coefficient of $G-\operatorname{deg}(\mathscr{F}, \Omega)$ can be easily described using amalgamated notation, for example

gap $>$ Print (AmalgamationSymbol( $\operatorname{ccs}[45])$ );

In this way we get the following description of some orbit types represented in $G-\operatorname{deg}(\mathscr{F}, \Omega)$ :

$$
\begin{aligned}
H_{4} & =-\left(D_{1} \times_{\mathbb{Z}_{2}} D_{1}^{z}\right), \quad H_{6}=\left(D_{1} \times \mathbb{Z}_{1}\right), \\
H_{8} & =\left(\mathbb{Z}_{1} \times D_{1}\right), \quad H_{18}=\left(D_{1} \times D_{1}\right), \\
H_{22} & =\left(\mathbb{Z}_{1} \times D_{3}\right), \quad H_{21}=\left(D_{1} \times \mathbb{Z}_{3}\right), \\
H_{43} & =\left(D_{3} \times D_{1}\right), \quad H_{44}=\left(D_{1} \times D_{3}\right), \\
H_{45} & =\left(D_{1} \times_{\mathbb{Z}_{2}}^{D_{3}} D_{3}^{p}\right), \quad H_{51}=\left(D_{3} \times D_{1}^{z}\right), \\
H_{63} & =\left(D_{3} \times D_{3}^{z}\right), \quad H_{67}=\left(D_{3} \times D_{3}\right) .
\end{aligned}
$$

Next we find the maximal orbit types in the representation $\mathbb{E}$ :

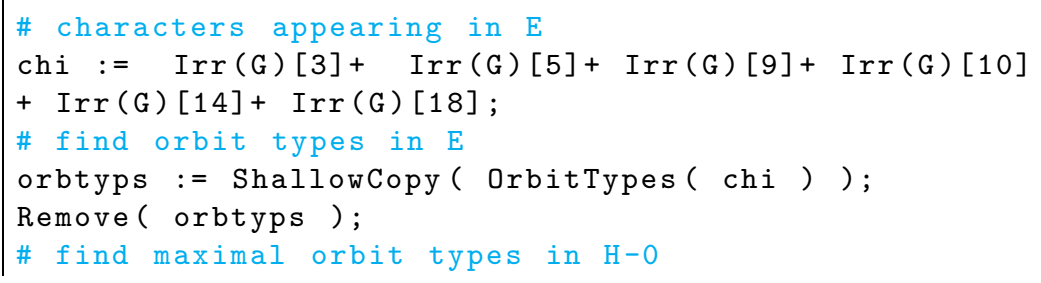


max_orbtyps := MaximalElements ( orbtyps );

Print ( List ( max_orbtyps, IdCCS ) );

The maximal orbit types in $\mathbb{E} \backslash\{0\}$ are

$$
\begin{array}{cc}
\left(H_{45}\right)=\left(D_{1} \times{ }_{\mathbb{Z}_{2}}^{D_{3}} D_{3}^{p}\right), & \left(H_{52}\right)=\left(D_{1} \times{ }_{\mathbb{Z}_{2}}^{D_{3}^{z}} D_{3}^{p}\right), \\
\left(H_{63}\right)=\left(D_{3} \times D_{3}^{z}\right), & \left(H_{67}\right)=\left(D_{3} \times D_{3}\right) .
\end{array}
$$

Consequently, we obtain the following

Theorem 2.14. Let $m=3$ and $\Gamma=D_{3}=\langle(1,2,3),(2,3)\rangle$ (acting on $\mathbb{R}^{3}$ by permuting the coordinates). Assume that $f: \mathbb{R} \times \mathbb{R}^{3} \rightarrow \mathbb{R}^{3}$ and $A$ (given by (34)) satisfy the conditions $\left(A_{1}\right)-\left(A_{6}\right)$. Then there exist

(i) at least one $\left(D_{1} \times_{\mathbb{Z}_{2}}^{D_{3}} D_{3}^{p}\right)$-orbit of (i.e. at least 6 different) $6 \pi$-periodic solutions to the system (1),

(ii) at least one $\left(D_{3} \times D_{3}^{z}\right)$-orbit of (i.e. at least 2 different) $6 \pi$-periodic solutions to the system (1),

(iii) at least one $\left(D_{3} \times D_{3}\right)$-orbit of (i.e. at least 2 different) $6 \pi$-periodic solutions to the system (1),

Therefore, the system (10) admits at least 10 different $6 \pi$-periodic solutions.

Case $m=4:$ In this case $G=D_{4} \times D_{3} \times \mathbb{Z}_{2}$, we have

$$
\sigma(\mathscr{A})=\left\{\lambda_{j, l}:=1+\frac{16\left(\mu_{l}-1\right)}{j^{2}+16}: l=0,1, j=0,1,2, \ldots\right\}
$$

Then

$$
\begin{gathered}
\sigma_{-}(\mathscr{A})=\left\{\lambda_{0,0}=-2, \lambda_{1,0}=-\frac{31}{17}, \lambda_{2,0}=-\frac{7}{5}, \lambda_{3,0}=-\frac{23}{25}, \lambda_{4,0}=-\frac{1}{2},\right. \\
\left.\lambda_{5,0}=-\frac{7}{41}, \lambda_{0,1}=-\frac{1}{2}, \lambda_{1,1}=-\frac{7}{17}, \lambda_{2,1}=-\frac{1}{5}\right\}
\end{gathered}
$$

each of the eigenvalues $\lambda_{l, i}$ are isotypicly simple, i.e. the eigenspaces $E\left(\lambda_{l, i}\right)$ are irreducible $G$-representations. More precisely, we have

$$
\begin{aligned}
& E\left(\lambda_{0,0}\right) \simeq \mathcal{V}_{0,0}^{-}, \quad E\left(\lambda_{4,0}\right) \simeq \mathcal{V}_{0,0}^{-} \oplus \mathcal{V}_{2,0}^{-}, \quad E\left(\lambda_{1,0}\right) \simeq E\left(\lambda_{3,0}\right) \simeq E\left(\lambda_{5,0}\right) \simeq \mathcal{V}_{0,1}^{-}, \\
& E\left(\lambda_{2,0}\right) \simeq \mathcal{V}_{3,0}^{-} \oplus \mathcal{V}_{4,0}^{-}, E\left(\lambda_{0,1}\right) \simeq \mathcal{V}_{0,1}^{-}, \quad E\left(\lambda_{1,1}\right) \simeq \mathcal{V}_{1,1}^{-}, E\left(\lambda_{2,1}\right) \simeq \mathcal{V}_{3,1}^{-} \oplus \mathcal{V}_{4,1}^{-}
\end{aligned}
$$

Therefore, we obtain

$$
\begin{aligned}
G-\operatorname{deg}(\mathscr{F}, \Omega)= & (G)-G-\operatorname{deg}\left(\mathscr{A}, B_{1}(0)\right) \\
= & (G)-\left(\operatorname{deg}_{\mathcal{V}_{0,0}^{-}}\right)^{2} \circ\left(\operatorname{deg}_{\mathcal{V}_{2,0}^{-}}\right) \circ\left(\operatorname{deg}_{\mathcal{V}_{0,1}^{-}}\right)^{3} \circ\left(\operatorname{deg}_{\mathcal{V}_{3,0}^{-}}\right) \circ\left(\operatorname{deg}_{\mathcal{V}_{4,0}^{-}}\right) \\
& \circ \operatorname{deg}_{\mathcal{V}_{0,2}^{-}} \circ \operatorname{deg}_{\mathcal{V}_{1,0}^{-}} \circ \operatorname{deg}_{\mathcal{V}_{1,1}^{-}} \circ \operatorname{deg}_{\mathcal{V}_{3,1}^{-}} \circ\left(\operatorname{deg}_{\mathcal{V}_{4,1}^{-}}\right) \\
= & (G)-\left(\operatorname{deg}_{\mathcal{V}_{2,0}^{-}}\right) \circ\left(\operatorname{deg}_{\mathcal{V}_{0,1}^{-}}\right) \circ\left(\operatorname{deg}_{\mathcal{V}_{3,0}^{-}}\right) \circ\left(\operatorname{deg}_{\mathcal{V}_{4,0}^{-}}\right) \circ \operatorname{deg}_{\mathcal{V}_{0,2}^{-}} \\
& \circ \operatorname{deg}_{\mathcal{V}_{1,0}^{-}} \circ \operatorname{deg}_{\mathcal{V}_{1,1}^{-}} \circ \operatorname{deg}_{\mathcal{V}_{3,1}^{-}} \circ\left(\operatorname{deg}_{\mathcal{V}_{4,1}^{-}}\right)
\end{aligned}
$$

GAP Code: $G:=D_{4} \times D_{3} \times \mathbb{Z}_{2}$.

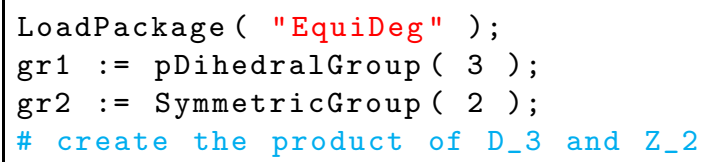




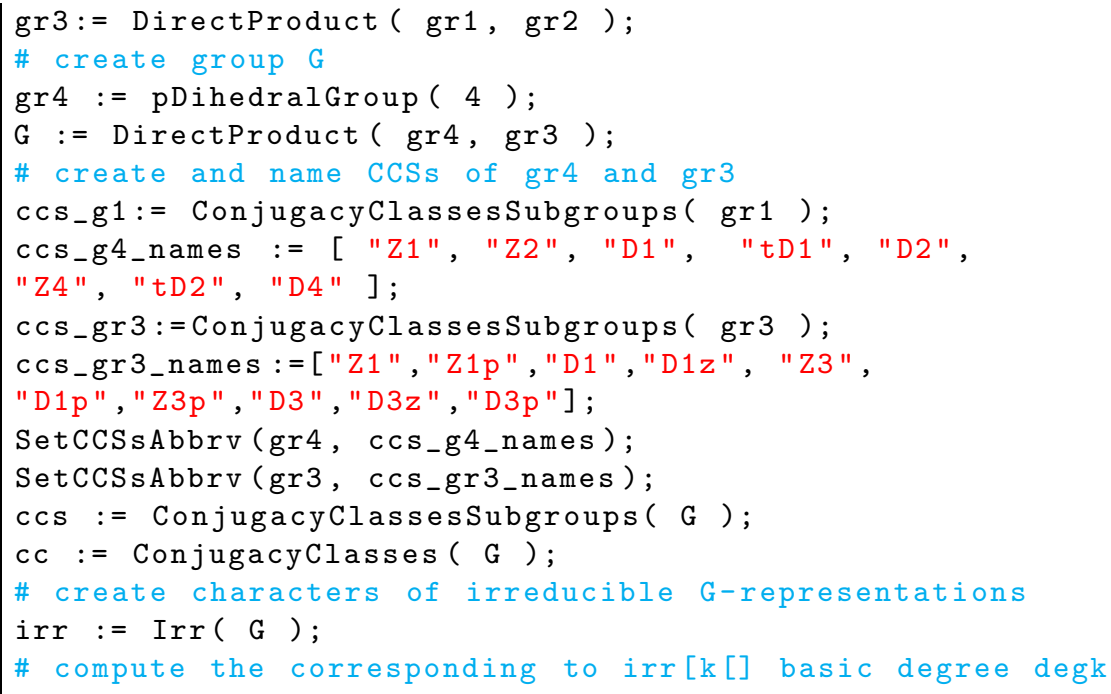

For a subgroup $K \leq D_{n}$, we use the symbol $K^{p}:=K \times \mathbb{Z}_{2}$ and in the code we simply write Kp. The group $G=D_{4} \times D_{3} \times \mathbb{Z}_{2}$ has 236 conjugacy classes of subgroups. The conjugacy classes are denoted $\left(H_{k}\right), k=1,2, \ldots, 236$, and are according to the same order as it is generated by GAP. The group G has 30 irreducible representations which can be easily identified in GAP.

\begin{tabular}{|l|l|l|}
\hline $\operatorname{Irr}(G)[2]=\chi_{\mathcal{V}_{2,2}^{-}}$ & $\operatorname{Irr}(G)[7]=\chi_{\mathcal{V}_{3,0}^{-}}$ & $\operatorname{Irr}(G)[19]=\chi_{\mathcal{V}_{4,1}^{-}}$ \\
$\operatorname{Irr}(G)[3]=\chi_{\mathcal{V}_{3,2}^{-}}$ & $\operatorname{Irr}(G)[8]=\chi_{\mathcal{V}_{4,0}^{-}}$ & $\operatorname{Irr}(G)[20]=\chi_{\mathcal{V}_{0,1}^{-}}$ \\
$\operatorname{Irr}(G)[4]=\chi_{\mathcal{V}_{4,2}^{-}}$ & $\operatorname{Irr}(G)[9]=\chi_{\mathcal{V}_{0,0}^{-}}$ & $\operatorname{Irr}(G)[25]=\chi_{\mathcal{V}_{1,2}^{-}}$ \\
$\operatorname{Irr}(G)[5]=\chi_{\mathcal{V}_{0,2}^{-}}$ & $\operatorname{Irr}(G)[17]=\chi_{\mathcal{V}_{2,1}^{-}}$ & $\operatorname{Irr}(G)[26]=\chi_{\mathcal{V}_{1,0}^{-}}$ \\
$\operatorname{Irr}(G)[6]=\chi_{\mathcal{V}_{2,0}^{-}}$ & $\operatorname{Irr}(G)[18]=\chi_{\mathcal{V}_{3,1}^{-}}$ & $\operatorname{Irr}(G)[30]=\chi_{\mathcal{V}_{1,1}^{-}}$ \\
\hline
\end{tabular}

Therefore, we are set up to compute the $\operatorname{degree} G-\operatorname{deg}(\mathscr{F}, \Omega)$ :

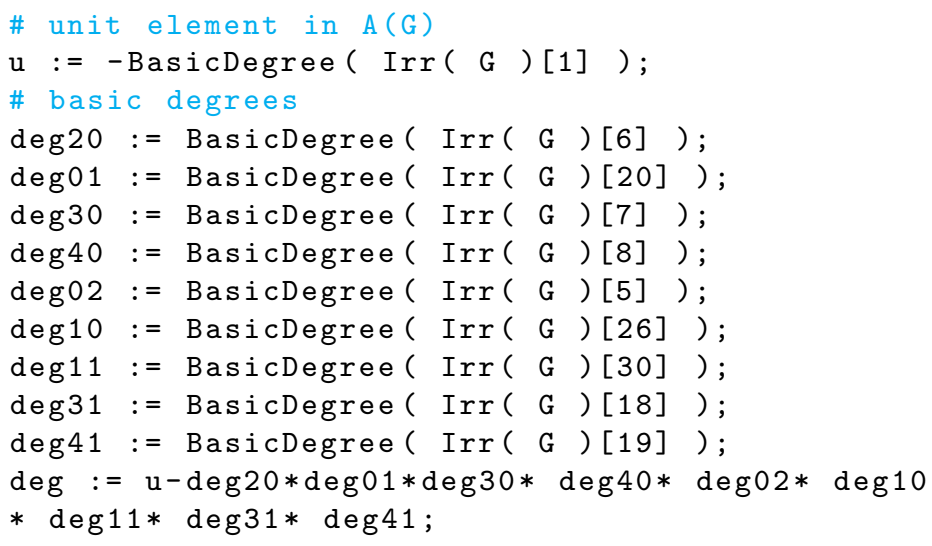

We also use the list of all irreducible $G$-representations generated by GAP. Using this list, the corresponding basic $G$-degrees are easily computed by the GAP program, so the exact 
value of $G-\operatorname{deg}(\mathscr{F}, \Omega)$ is given by

$$
\begin{aligned}
G-\operatorname{deg}(\mathscr{F}, \Omega) & =-3\left(H_{1}\right)+\left(H_{2}\right)+\left(H_{3}\right)+\left(H_{4}\right)-\left(H_{6}\right)+2\left(H_{8}\right)+\left(H_{9}\right) \\
& +\left(H_{10}\right)+2\left(H_{11}\right)-\left(H_{12}\right)+\left(H_{13}\right)+\left(H_{14}\right)-\left(H_{17}\right)+\left(H_{18}\right)-\left(H_{19}\right) \\
& -\left(H_{20}\right)-\left(H_{23}\right)-\left(H_{25}\right)-\left(H_{26}\right)+\left(H_{27}\right)-\left(H_{28}\right)-\left(H_{32}\right)+\left(H_{33}\right) \\
& -\left(H_{34}\right)+\left(H_{35}\right)-\left(H_{36}\right)-\left(H_{39}\right)+\left(H_{40}\right)-\left(H_{43}\right)-\left(H_{55}\right)+2\left(H_{57}\right) \\
& -\left(H_{60}\right)+\left(H_{62}\right)+\left(H_{68}\right)+\left(H_{71}\right)+\left(H_{72}\right)-\left(H_{73}\right)-\left(H_{76}\right)+\left(H_{86}\right) \\
& +\left(H_{87}\right)-\left(H_{88}\right)-\left(H_{90}\right)-\left(H_{92}\right)-\left(H_{95}\right)+\left(H_{99}\right)+\left(H_{100}\right)+\left(H_{102}\right) \\
& +\left(H_{103}\right)-\left(H_{104}\right)+\left(H_{105}\right)+\left(H_{109}\right)--\left(H_{116}\right)+\left(H_{117}\right)+\left(H_{119}\right)-\left(H_{120}\right) \\
& -\left(H_{122}\right)+\left(H_{130}\right)-\left(H_{135}\right)-\left(H_{138}\right)-\left(H_{144}\right)+\left(H_{148}\right)+\left(H_{151}\right)+\left(H_{167}\right) \\
& +\left(H_{169}\right)+\left(H_{170}\right)-m\left(H_{173}\right)+\left(H_{174}\right)+\left(H_{175}\right)+\left(H_{177}\right)+\left(H_{179}\right)-\left(H_{192}\right) \\
& -\left(H_{203}\right)-\left(H_{208}\right)-\left(H_{212}\right)-\left(H_{214}\right)+\left(H_{223}\right)+\left(H_{225}\right)+\left(H_{229}\right)+\left(H_{233}\right) .
\end{aligned}
$$

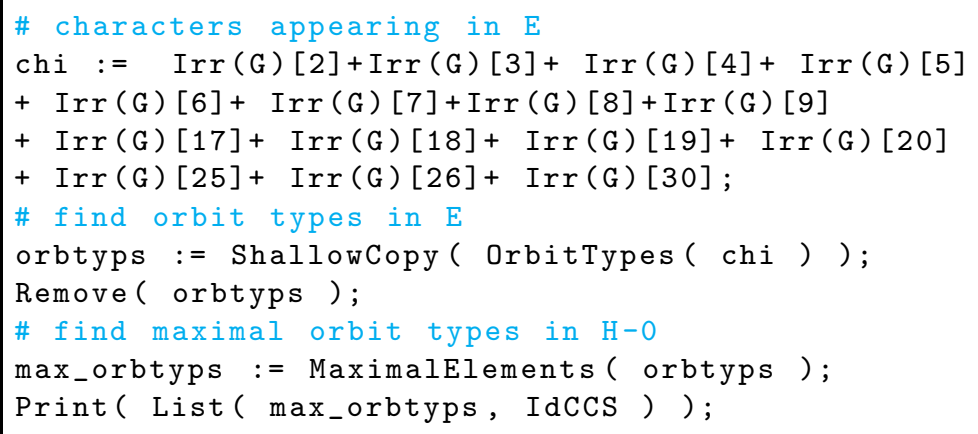

Since the $G$-isotypic components in $\mathbb{E}$ are easily identified, the GAP program also allows a quick computation of all maximal orbit types in $\mathbb{E} \backslash\{0\}$, namely

$$
\left(H_{177}\right),\left(H_{178}\right),\left(H_{179}\right),\left(H_{180}\right),\left(H_{223}\right),\left(H_{224}\right),\left(H_{225}\right),
$$

$\left(H_{228}\right), \quad\left(H_{229}\right), \quad\left(H_{232}\right), \quad\left(H_{233}\right)$.

One can notice that $G-\operatorname{deg}(\mathscr{F}, \Omega)$ has non-zero coefficients for the following maximal orbit types:

$$
\begin{aligned}
& \left(H_{177}\right)=\left(D_{2}^{D_{1}} \times_{\mathbb{Z}_{2}}^{D_{3}} D_{3}^{p}\right), \quad\left(H_{179}\right)=\left(\widetilde{D}_{2}^{\widetilde{D}_{1}} \times_{\mathbb{Z}_{2}}^{D_{3}} D_{3}^{p}\right) \\
& \left(H_{223}\right)=\left(D_{4} \times D_{3}^{z}\right), \quad\left(H_{225}\right)=\left(D_{4}^{D_{2}} \times_{\mathbb{Z}_{2}}^{D_{3}} D_{3}^{p}\right), \\
& \left(H_{229}\right)=\left(D_{4}^{D_{2}} \times_{\mathbb{Z}_{2}}^{D_{3}} D_{3}^{p}\right),\left(H_{233}\right)=\left(D_{4}^{\mathbb{Z}_{4}} \times_{\mathbb{Z}_{2}}^{D_{3}} D_{3}^{p}\right)
\end{aligned}
$$

Consequently, we obtain the following result.

Theorem 2.15. Let $m=4, k=3$ and $\Gamma=D_{3}=\langle(1,2,3),(2,3)\rangle$ (acting on $\mathbb{R}^{3}$ by permuting the coordinates). Assume that $f: \mathbb{R} \times \mathbb{R}^{3} \rightarrow \mathbb{R}^{3}$ and $A$ (given by (34)) satisfy the conditions $\left(A_{1}\right)-\left(A_{6}\right)$. Then there exist

(i) at least one $\left(D_{2}^{D_{1}} \times_{\mathbb{Z}_{2}}^{D_{3}} D_{3}^{p}\right)$-orbit of (i.e. at least 4 different) $8 \pi$-periodic solutions to the system (1),

(ii) at least one $\left(\widetilde{D}_{2}^{\widetilde{D}_{1}} \times_{\mathbb{Z}_{2}}^{D_{3}} D_{3}^{p}\right)$-orbit of (i.e. at least 4 different) $8 \pi$-periodic solutions to the system (1),

(iii) at least one $\left(D_{4} \times D_{3}^{z}\right)$-orbit of (i.e. at least 2 different) $8 \pi$-periodic solutions to the system (1), 
(iv) at least one $\left(D_{4}^{D_{2}} \times_{\mathbb{Z}_{2}}^{D_{3}} D_{3}^{p}\right)$-orbit of (i.e. at least 2 different) $8 \pi$-periodic solutions to the system (1),

(v) at least one $\left(D_{4}^{D_{2}} \times_{\mathbb{Z}_{2}}^{D_{3}} D_{3}^{p}\right)$-orbit of (i.e. at least 2 different) $8 \pi$-periodic solutions to the system (1),

(vi) at least one $\left(D_{4}^{\mathbb{Z}_{4}} \times{ }_{\mathbb{Z}_{2}}^{D_{3}} D_{3}^{p}\right)$-orbit of (i.e. at least 2 different) $8 \pi$-periodic solutions to the system (1).

Therefore, the system (11) admits at least 16 different $8 \pi$-periodic solutions.

\section{Bifurcation in Reversible Non-Autonomous Sec- ond Order Differential Equaitons}

Consider the following parametrized system with $2 \pi$-periodic coefficients

$$
\ddot{u}(t)=(-\alpha \operatorname{Id}+A) u(t)+f(t, u(t)), u(t) \in \mathbb{R}^{k},
$$

where $A$ is a non-singular $k \times k$-matrix and $f: \mathbb{R} \times \mathbb{R}^{k} \rightarrow \mathbb{R}^{k}$ is a continuous function satisfying the conditions $\left(A_{1}\right)-\left(A_{3}\right)$ and :

$\left(B_{4}\right) \quad \lim _{x \rightarrow 0} \frac{f(t, x)}{|x|}=0$ uniformly with respect to $t$.

We are interested in studying the bifurcation of the subharmonic $2 \pi m$-periodic solutions (for some integer $m$ ) to (28) from the trivial solution $(\alpha, 0)$, i.e. the solutions

$$
u(t)=u(t+2 \pi m), \quad \dot{u}(t)=\dot{u}(t+2 \pi m) .
$$

We also consider a subgroup $\Gamma \leq S_{k}$ which acts on $V:=\mathbb{R}^{k}$ by permuting the coordinates of vectors $x=\left(x_{1}, x_{2}, \ldots, x_{k}\right)^{T}$ in $\mathbb{R}^{k}$ given by

$$
\sigma x=\sigma\left(x_{1}, x_{2}, \ldots, x_{k}\right)^{T}:=\left(x_{\sigma(1)}, x_{\sigma(2)}, \ldots, x_{\sigma(k)}\right)^{T} .
$$

Clearly, the space $V:=\mathbb{R}^{k}$ equipped with this $\Gamma$-action is an orthogonal $\Gamma$-representation. We also introduce the following conditions

$\left(B_{5}\right) \quad$ For all $t \in \mathbb{R}, x \in V$ and $\sigma \in \Gamma$, we have $f(t, \sigma x)=\sigma f(t, x)$ and $A \sigma x=\sigma A x$;

The condition $\left(B_{5}\right)$ implies that the system (28) is $\Gamma$-symmetric. The bifurcation problem (28) with the boundary conditions (29) can be expressed as the following equation

$$
\mathscr{F}(\alpha, u)=0, \quad(\alpha, u) \in \mathbb{R} \oplus \mathbb{E},
$$

where

$$
\mathscr{F}(\alpha, u):=u-L^{-1}\left(N_{A+f}(\mathfrak{j}(u))-(\alpha+1) \mathfrak{j}(u)\right), \quad \alpha \in \mathbb{R}, u \in \mathbb{E} .
$$

Put $G:=\Gamma \times D_{m} \times \mathbb{Z}_{2}$. Notice that under the assumptions $\left(A_{1}\right)-\left(A_{3}\right)$ and $\left(B_{5}\right)$, the map $\mathscr{F}$ is $G$-equivariant completely continuous field such that $\mathscr{F}(\alpha, 0)=0$ for all $\alpha \in \mathbb{R}$. Moreover, the assumption $\left(B_{4}\right)$ implies that $\mathscr{F}$ is differentiable at $(\alpha, 0)$ with respect to $u \in \mathbb{E}$, and

$$
\mathscr{A}(\alpha):=D_{u} \mathscr{F}(\alpha, 0)=\mathrm{Id}-L^{-1}\left(N_{A} \circ \mathrm{j}-(\alpha+1) \mathrm{j}\right): \mathbb{E} \rightarrow \mathbb{E} .
$$

The necessary condition for the point $\left(\alpha_{o}, 0\right)$ to be a bifurcation point for (31) is that $\mathscr{A}\left(\alpha_{o}\right)$ : $\mathbb{E} \rightarrow \mathbb{E}$ is not an isomorphism, i.e. $0 \in \sigma\left(\mathscr{A}\left(\alpha_{o}\right)\right.$.

The point $\alpha_{o}$ is called a critical point for (31) and the set of all such critical points $\alpha_{o}$ is denoted $\Lambda$. One can easily compute the spectrum of the operator $\mathscr{A}\left(\alpha_{o}\right)$ :

$$
\sigma\left(\mathscr{A}\left(\alpha_{o}\right)\right):=\left\{1+\frac{m^{2}\left(\mu-\alpha_{o}-1\right)}{j^{2}+m^{2}}: j=0,1,2, \ldots, \mu \in \sigma(A)\right\}
$$


which implies that

$$
\Lambda=\left\{\alpha_{j, \mu}:=\frac{j^{2}+m^{2} \mu}{m^{2}}: j=0,1,2, \ldots, \mu \in \sigma(A)\right\} .
$$

\subsection{Bifurcation in System (28) without Symmetries}

Assume that $\Gamma=\{e\}$, i.e. $G:=D_{m} \times \mathbb{Z}_{2}$, and that $\alpha_{j, \mu} \neq \alpha_{j^{\prime}, \mu^{\prime}}$ for $(j, \mu) \neq\left(j^{\prime}, \mu^{\prime}\right)$. Let us put all the elements of $\Lambda$ in increasing order, i.e. $\cdots<\alpha_{j_{k}, \mu_{k}}<\alpha_{j_{k+1}, \mu_{k+1}}<\ldots$ Then for every $\alpha_{j_{o}, \mu_{o}} \in \Lambda$ we have

$$
\omega_{G}\left(\alpha_{j_{o}, \mu_{o}}\right)=\prod_{\alpha_{j_{k}, \mu_{k}}<\alpha_{j_{o}, \mu_{o}}}\left(\operatorname{deg}_{\mathcal{W}_{i\left(j_{k}\right)}^{-}}\right)^{m\left(\mu_{k}\right)}\left((G)-\left(\operatorname{deg}_{\mathcal{W}_{i\left(j_{o}\right)}^{-}}\right)^{m\left(\mu_{o}\right)}\right) .
$$

Theorem 3.1. Suppose $A: V \rightarrow V$ and $f: \mathbb{R} \times V \rightarrow V$ satisfies the assumptions ( $\left.A_{1}\right)$ $\left(A_{3}\right)$ and $\left(B_{4}\right)$ and let $\Lambda$ be the critical set for (31). Assume that for all $\alpha_{j, \mu}, \alpha_{j^{\prime}, \mu^{\prime}} \in \Lambda$ we have $\alpha_{j, \mu} \neq \alpha_{j^{\prime}, \mu^{\prime}}$ if $(j, \mu) \neq\left(j^{\prime}, \mu^{\prime}\right)$. Then for every $\alpha_{j_{o}, \mu_{o}} \in \Lambda$ such that $m\left(\mu_{o}\right)$ is odd we have $\omega\left(\alpha_{j_{o}, \mu_{o}}\right) \neq 0$, i.e. the point $\left(\alpha_{j_{o}, \mu_{o}}, 0\right)$ is a bifurcation point of non-trivial $2 \pi m$-periodic solutions for (28).

Proof. Notice that under the assumption that $m\left(\mu_{o}\right)$ is odd, we have

$$
(G)-\left(\operatorname{deg}_{\mathcal{W}_{i\left(j_{o}\right)}^{-}}\right)^{m\left(\mu_{o}\right)}=(G)-\left(\operatorname{deg}_{\mathcal{W}_{i\left(j_{o}\right)}^{-}}\right) \neq 0 .
$$

Since the product $\prod_{\alpha_{j_{k}, \mu_{k}}<\alpha_{j_{o}, \mu_{o}}}\left(\operatorname{deg}_{\mathcal{W}_{i\left(j_{k}\right)}^{-}}\right)^{m\left(\mu_{k}\right)}$ is an invertible element in $A(G)$, it follows from formula (33) that $\omega_{G}\left(\alpha_{j_{o}, \mu_{o}}\right)$ is non-zero. Therefore, by TheoremB.1 the point $\left(\alpha_{j_{o}, \mu_{o}}, 0\right)$ is a bifurcation point of non-trivial $2 \pi m$-periodic solutions for (28).

Consequently, we obtain the following:

Theorem 3.2. Suppose $A: V \rightarrow V$ and $f: \mathbb{R} \times V \rightarrow V$ satisfies the assumptions $\left(A_{1}\right)-\left(A_{3}\right)$ and $\left(B_{4}\right)$. Assume that $k>0$ is odd and $\sigma(A)$ consists of exactly $k$ different eigenvalues such that for all $(j, \mu) \neq\left(j^{\prime}, \mu^{\prime}\right)$ the critical points $\alpha_{j, \mu}, \alpha_{j^{\prime}, \mu^{\prime}} \in \Lambda$ are also different. Suppose $m=2^{\varepsilon_{0}} p_{1}^{\varepsilon_{1}} p_{2}^{\varepsilon_{2}} \ldots p_{s}^{\varepsilon_{s}}$, where $\varepsilon_{0} \geq 0, \varepsilon_{l}>0$ and $p_{l}$ are the prime numbers such that $2 \leq p_{1}<$ $p_{2}<\cdots<p_{s}$. For $l=1,2, \ldots, s$ put $m_{l}:=\frac{m}{p_{l}}$. Then

(a) if $p_{l}>2$ and $\rho_{l}$ is odd, then the system (31) admits an unbounded branch of $2 \pi m$-periodic solutions with symmetries at least $\left(D_{m_{l}}^{z}\right), m_{l}:=\frac{m}{p_{l}}$, and

(b) if $p_{1}=2, \varepsilon_{1}=1$, and $\rho_{1}$ is odd, then the system (31) admits an unbounded branch of $2 \pi m$-periodic solutions with symmetries at least $\left(D_{m}^{d}\right),\left(D_{m}^{\hat{d}}\right)$, and

(c) if $p_{1}=2$ and $\varepsilon_{1}>1$, then and $\rho_{l}$ is odd, then the system (31) admits an unbounded branch of $2 \pi m$-periodic solutions with symmetries at least $\left(D_{\frac{m}{2}}^{d}\right),\left(\widetilde{D}_{\frac{m}{2}}^{d}\right)$.

\subsection{Bifurcation in System (28) with Additional Symmetries $\Gamma$}

In this section we assume that $k=3, f: \mathbb{R} \times \mathbb{R}^{3} \rightarrow \mathbb{R}^{3}$ satisfies assumptions $\left(A_{1}\right)-\left(A_{3}\right)$ and $\left(B_{4}\right)-\left(B_{5}\right)$ with $\Gamma=D_{3}$ and

$$
A=\frac{1}{4}\left[\begin{array}{lll}
-4 & -2 & -2 \\
-2 & -4 & -2 \\
-2 & -2 & -4
\end{array}\right]
$$

Then we have

$$
\sigma(A)=\left\{\mu_{0}=-2, \mu_{1}=-\frac{1}{2}\right\}
$$


Case $m=3: \quad$ In this case $G=D_{3} \times D_{3} \times \mathbb{Z}_{2}$ and we have

$$
\sigma(\mathscr{A}(\alpha))=\left\{\lambda_{j, l}:=1+\frac{9\left(\mu_{l}-\alpha-1\right)}{j^{2}+9}: l=0,1, j=0,1,2, \ldots\right\}
$$

Then

$$
\begin{gathered}
\Lambda=\left\{\alpha_{0,0}=-2, \alpha_{0,1}=-1 / 2, \alpha_{1,0}=-\frac{17}{9}, \alpha_{1,1}=-\frac{7}{18}, \ldots,\right. \\
\left.\alpha_{j, 0}=\frac{j^{2}-18}{9}, \alpha_{j, 1}=\frac{j^{2}-9 / 2}{9}, \ldots\right\},
\end{gathered}
$$

and each of the critical values $\alpha_{j, i}$ is isotypicly simple, i.e. the eigenspace $E\left(\lambda_{j, i}\right)$ is an irreducible $G$-representation $\mathcal{V}_{i(j), l}^{-}$. Maximal orbit types in $\mathbb{E} \backslash\{0\}$ are listed in (27). Using the same GAP code as in Example (for $D_{3}$ ) in subsection 2.7, we can compute the exact bifurcation invariants. Indeed, we have the following critical points from $\Lambda$

$$
\alpha_{0,0}<\alpha_{1,0}<\alpha_{2,0}<\alpha_{0,1}<\alpha_{1,1}<\alpha_{2,1}<\alpha_{3,0}<\alpha_{3,1}<\alpha_{4,0}<\alpha_{4,1}<\alpha_{5,1}<\ldots
$$

Thus

$$
\begin{aligned}
\omega\left(\alpha_{0,0}\right) & =(G)-\operatorname{deg}_{\mathcal{V}_{0,0}^{-}}=\left(H_{67}\right) \\
\omega\left(\alpha_{1,0}\right) & =\operatorname{deg}_{\mathcal{V}_{0,0}^{-}} \circ\left((G)-\operatorname{deg}_{\mathcal{V}_{1,0}^{-}}\right)=-\left(H_{43}\right)+\left(H_{51}\right) \\
\omega\left(\alpha_{2,0}\right) & =\operatorname{deg}_{\mathcal{V}_{0,0}^{-}} \circ \operatorname{deg}_{\mathcal{V}_{1,0}^{-}} \circ\left((G)-\operatorname{deg}_{\mathcal{V}_{1,0}^{-}}\right)=\left(H_{43}\right)-\left(H_{51}\right) \\
\omega\left(\alpha_{0,1}\right) & =\operatorname{deg}_{\mathcal{V}_{0,0}^{-}} \circ\left((G)-\operatorname{deg}_{\mathcal{V}_{0,0}^{-}} \circ \operatorname{deg}_{\mathcal{V}_{2,0}^{-}}=\left(H_{63}\right)+\left(H_{67}\right)\right) \\
\omega\left(\alpha_{1,1}\right) & =\operatorname{deg}_{\mathcal{V}_{2,0}^{-}} \circ\left((G)-\operatorname{deg}_{\mathcal{V}_{1,1}^{-}}\right)=\left(H_{1}\right)-2\left(H_{3}\right)+\left(H_{4}\right)-\left(H_{5}\right)-\left(H_{8}\right)+\left(H_{13}\right) \\
& +\left(H_{14}\right)-\left(H_{16}\right)+\left(H_{18}\right)-\left(H_{28}\right)+\left(H_{31}\right) \\
\omega\left(\alpha_{4,0}\right) & =\operatorname{deg}_{\mathcal{V}_{2,0}^{-}} \circ \operatorname{deg}_{\mathcal{V}_{1,1}^{-}} \circ\left((G)-\operatorname{deg}_{\mathcal{V}_{1,0}^{-}}\right)=\left(H_{3}\right)-\left(H_{4}\right)-\left(H_{7}\right) \\
& +\left(H_{8}\right)+2\left(H_{16}\right)-2\left(H_{18}\right)+\left(H_{43}\right)-\left(H_{51}\right) \\
\omega\left(\alpha_{2,1}\right) & =\operatorname{deg}_{\mathcal{V}_{2,0}^{-}} \circ \operatorname{deg}_{\mathcal{V}_{1,1}^{-}} \circ \operatorname{deg}_{\mathcal{V}_{1,0}^{-}} \circ\left((G)-\operatorname{deg}_{\mathcal{V}_{1,1}^{-}}\right)=-\left(H_{1}\right)+\left(H_{3}\right)+\left(H_{5}\right) \\
& +\left(H_{7}\right)-\left(H_{13}\right)-\left(H_{14}\right)-\left(H_{16}\right)+\left(H_{18}\right)+\left(H_{28}\right)-\left(H_{31}\right)
\end{aligned}
$$

\section{A APPENDIX: $G$-Equivariant Brouwer Degree}

Equivariant Notation: For a subgroup $H$ of $G$, i.e. $H \leq G$, we denote the normalizer of $H$ in $G$ by $N(H)$ and the Weyl group of $H$ by $W(H)=N(H) / H$. The symbol $(H)$ stands for the conjugacy class of $H$ in $G$. We put $\Phi(G):=\{(H): H \leq G\}$, i.e. $\Phi(G)$ is the set of conjugacy classes of subgroups in $G$. $\Phi(G)$ has a natural partial order defined by $(H) \leq(K)$ iff $\exists g \in G g \mathrm{Hg}^{-1} \leq K$. For two subgroups $L, H \leq G$, we denote by $n(L, H)$ the number of different subgroups $H^{\prime}$ conjugate to $H$ such that $L \leq H^{\prime}$.

For a $G$-space $X$ and $x \in X$, we denote the isotropy group of $x$ by $G_{x}:=\{g \in G: g x=x\}$, the orbit of $x$ by $G(x):=\{g x: g \in G\}$, and we call $\left(G_{x}\right)$ the orbit type of $x \in X$. For a subgroup $H \leq G$ the subspace $X^{H}:=\left\{x \in X: G_{x} \geq H\right\}$ is called the $H$-fixed-point subspace of $X$. Clearly, $W(H)$ acts on $X^{H}$.

Consider a complete list of all irreducible $G$-representations $\mathcal{V}_{i}, i=0,1, \ldots, r$. Such list for concrete group $G$ can be established by using GAP. Let $V$ be a finite-dimensional 
$G$ - representation and (without loss of generality) we may assume that $V$ is an orthogonal representation. Then $V$ decomposes into a direct sum

$$
V=V_{0} \oplus V_{1} \oplus \cdots \oplus V_{r}
$$

where each component $V_{i}$ is modeled on the irreducible $G$-representation $\mathcal{V}_{i}, i=0,1,2, \ldots, r$, that is $V_{i}$ contains all the irreducible subrepresentations of $V$ equivalent to $\mathcal{V}_{i}$. The decomposition (35) is called $G$-isotypic decomposition of $V$. Denote the $\mathbb{R}$-algebra (resp. group) of all $G$-equivariant linear (resp. invertible) operators on $V$ by $\mathrm{L}^{G}(V)$ (resp. GL ${ }^{G}(V)$ ) . Clearly, the isotypic decomposition (35) induces the following direct sum decomposition of $\mathrm{GL}^{G}(V)$ :

$$
\mathrm{GL}^{G}(V)=\bigoplus_{i=0}^{r} \mathrm{GL}^{G}\left(V_{i}\right)
$$

where for every isotypic component $V_{i}$

$$
\mathrm{GL}^{G}\left(V_{i}\right) \simeq \mathrm{GL}\left(m_{i}, \mathbb{F}\right), \quad m_{i}=\operatorname{dim} V_{i} / \operatorname{dim} \mathcal{V}_{i}
$$

and depending on the type of the irreducible representation $\mathcal{V}_{i}, \mathbb{F}(=\mathbb{R}, \mathbb{C}$ or $\mathbb{H})$ is a finitedimensional division algebra $\mathrm{L}^{G}\left(\mathcal{V}_{i}\right)$.

\section{A.1 Burnside Ring}

We assume that $G$ is a finite group. Denote the free abelian group generated by $(H) \in \Phi(G)$ by $A(G):=\mathbb{Z}[\Phi(G)]$, i.e., an element $a \in A(G)$ can be written as a sum

$$
a=n_{1}\left(H_{1}\right)+\cdots+n_{m}\left(H_{m}\right)
$$

where $n_{i} \in \mathbb{Z}$ and $\left(H_{i}\right) \in \Phi(G)$. There is a natural multiplication operation $\circ: A(G) \times A(G) \rightarrow$ $A(G)$ which is defined on generators $(H),(K) \in \Phi(G)$ by

$$
(H) \cdot(K)=\sum_{(L) \in \Phi(G)} m_{L}(L),
$$

where the integer $m_{L}$ represents the number of $(L)$-orbits contained in the space $G / H \times G / K$. The numbers $m_{L}$ can be easily computed from the following recurrence formula

$$
m_{L}=\frac{n(L, H)|W(H)| n(L, K)|W(K)|-\sum_{(\widetilde{L})>(L)} m_{\widetilde{L}} n(L, \widetilde{L})|W(\widetilde{L})|}{|W(L)|} .
$$

Together with the multiplication ' $\circ$ ', $A(G)$ becomes a ring with the unity $(G)$, which is called the Burnside ring of $G$. For more details see [2]

\section{A.2 Axioms of Brouwer G-Equivariant Degree}

Consider an orthogonal $G$-representation $V$, a continuous $G$-map $f: V \rightarrow V$, and an open bounded $G$-invariant set $\Omega \subset V$ such that for all $x \in \partial \Omega$, we have $f(x) \neq 0$. Then $f$ is called $\Omega$-admissible and $(f, \Omega)$ is called a $G$-admissible pair (in $V$ ). The set of all possible $G$-pairs will be denoted by $\mathcal{M}^{G}$.

The following result (cf [2]) can be considered as an axiomatic definition of the G-equivariant Brouwer degree:

Theorem A.1. There exists a unique map $G$ - $\operatorname{deg}: \mathcal{M}^{G} \rightarrow A(G)$, which assigns to every admissible $G$-pair $(f, \Omega)$ an element $G-\operatorname{deg}(f, \Omega) \in A(G)$

$$
G-\operatorname{deg}(f, \Omega)=\sum_{(H)} n_{H}(H)=n_{H_{1}}\left(H_{1}\right)+\cdots+n_{H_{m}}\left(H_{m}\right),
$$

satisfying the following properties: 
- (Existence) If $G-\operatorname{deg}(f, \Omega) \neq 0$, i.e., $n_{H_{i}} \neq 0$ for some $i$ in (39), then there exists $x \in \Omega$ such that $f(x)=0$ and $\left(G_{x}\right) \geq\left(H_{i}\right)$.

- (Additivity) Let $\Omega_{1}$ and $\Omega_{2}$ be two disjoint open $G$-invariant subsets of $\Omega$ such that $f^{-1}(0) \cap \Omega \subset \Omega_{1} \cup \Omega_{2}$. Then

$$
G-\operatorname{deg}(f, \Omega)=G-\operatorname{deg}\left(f, \Omega_{1}\right)+G-\operatorname{deg}\left(f, \Omega_{2}\right) .
$$

- (Homotopy) If $h:[0,1] \times V \rightarrow V$ is an $\Omega$-admissible $G$-homotopy, then

$$
G-\operatorname{deg}\left(h_{t}, \Omega\right)=\text { constant. }
$$

- (Normalization) Let $\Omega$ be a G-invariant open bounded neighborhood of 0 in $V$. Then

$$
G-\operatorname{deg}(\operatorname{Id}, \Omega)=(G) .
$$

- (Multiplicativity) For any $\left(f_{1}, \Omega_{1}\right),\left(f_{2}, \Omega_{2}\right) \in \mathcal{M}^{G}$,

$$
G-\operatorname{deg}\left(f_{1} \times f_{2}, \Omega_{1} \times \Omega_{2}\right)=G-\operatorname{deg}\left(f_{1}, \Omega_{1}\right) \circ G-\operatorname{deg}\left(f_{2}, \Omega_{2}\right),
$$

where the multiplication ' $\mathrm{O}$ ' is taken in the Burnside ring $A(G)$.

- (Suspension) If $W$ is an orthogonal $G$-representation and $\mathscr{B}$ is an open bounded invariant neighborhood of $0 \in W$, then

$$
G-\operatorname{deg}\left(f \times \operatorname{Id}_{W}, \Omega \times \mathscr{B}\right)=G-\operatorname{deg}(f, \Omega) .
$$

- (Recurrence Formula) For an admissible $G$-pair $(f, \Omega)$, the $G$-degree (39) can be computed using the following recurrence formula

$$
n_{H}=\frac{\operatorname{deg}\left(f^{H}, \Omega^{H}\right)-\sum_{(K)>(H)} n_{K} n(H, K)|W(K)|}{|W(H)|},
$$

where $|X|$ stands for the number of elements in the set $X$ and $\operatorname{deg}\left(f^{H}, \Omega^{H}\right)$ is the Brouwer degree of the map $f^{H}:=\left.f\right|_{V H}$ on the set $\Omega^{H} \subset V^{H}$.

$\Omega$.

The $G$-deg $(f, \Omega)$ is called the $G$-equivariant Brouwer degree (or simply $G$-degree) of $f$ in

\section{A.3 Computation of $\Gamma$-Equivariant Degree}

Put $B(V):=\{x \in V:|x|<1\}$. For each irreducible $G$-representation $\mathcal{V}_{i}, i=0,1,2, \ldots$, we define

$$
\operatorname{deg}_{\mathcal{V}_{i}}:=G-\operatorname{deg}\left(-\operatorname{Id}, B\left(\mathcal{V}_{i}\right)\right),
$$

and will call $\operatorname{deg}_{\mathcal{V}_{i}}$ the basic degree.

Consider a $G$-equivariant linear isomorphism $T: V \rightarrow V$ and assume that $V$ has a $G$ isotypic decomposition (35). Then by the Multiplicativity property, we have

$$
G-\operatorname{deg}(T, B(V))=\prod_{i=0}^{r} G-\operatorname{deg}\left(T_{i}, B\left(V_{i}\right)\right)=\prod_{i=0}^{r} \prod_{\mu \in \sigma_{-}(T)}\left(\operatorname{deg}_{\mathcal{V}_{i}}\right)^{m_{i}(\mu)}
$$

where $T_{i}=\left.T\right|_{V_{i}}$ and $\sigma_{-}(T):=\{\mu \in \sigma(T): \mu<0\}$ denotes the real negative spectrum of $T$.

Notice that the basic degrees can be effectively computed from (40), i.e. formula

$$
\operatorname{deg}_{\mathcal{V}_{i}}=\sum_{(H)} n_{H}(H)
$$

where

$$
n_{H}=\frac{(-1)^{\operatorname{dim} \mathcal{V}_{i}^{H}}-\sum_{H<K} n_{K} n(H, K)|W(K)|}{|W(H)|} .
$$




\section{B Local and Global Bifurcation Problems}

Assume that $a<b, V$ is an orthogonal $G$-representation and $\Omega \subset V$ is an open bounded $G$-invariant subset. Let $f: \mathbb{R} \oplus V \rightarrow V$ be a continuous $G$-equivariant map such that $\left(f_{a}, \Omega\right)$, $\left(f_{b}, \Omega\right) \in \mathcal{M}^{G}(V)$, where $f_{t}(x):=f(t, x), t \in \mathbb{R}, x \in V$. Then a continuous $G$-invariant function $\varphi: \mathbb{R} \oplus V \rightarrow \mathbb{R}$ will be called $\Omega$-complementing function for $f_{t}$ at $t=a, b$, if

$$
\begin{cases}\varphi(t, x)<0 & \text { if } t=a, b, x \in \Omega \\ \varphi(t, x)>0 & \text { if } t=(a, b), x \in \partial \Omega\end{cases}
$$

In such a case we define the map $F_{\varphi}: \mathbb{R} \oplus V \rightarrow \mathbb{R} \oplus V$ by

$$
F_{\varphi}(t, x)=(\varphi(t, x), f(t, x)), \quad t \in \mathbb{R}, x \in V .
$$

The following result is well-known in non-equivariant case.

Theorem B.1. Suppose that $f: \mathbb{R} \oplus V \rightarrow V$ is a $G$-equivariant map such that $\left(f_{a}, \Omega\right)$, $\left(f_{b}, \Omega\right) \in \mathcal{M}^{G}(V)$, and $\varphi: \mathbb{R} \oplus V \rightarrow V$ is an $\Omega$-complementing function for $f_{t}$ at $t=a, b$. Then $\left(F_{\varphi},(a, b) \times \Omega\right) \in \mathcal{M}^{G}$, the $G$-equivariant degree $G$-deg $\left(F_{\varphi},(a, b) \times \Omega\right)$ doesn't depend on the choice of the $\Omega$-complementing function $\varphi$ and we have

$$
G-\operatorname{deg}\left(F_{\varphi},(a, b) \times \Omega\right)=G-\operatorname{deg}\left(f_{a}, \Omega\right)-G-\operatorname{deg}\left(f_{b}, \Omega\right) .
$$

Let $f: \mathbb{R} \times V \rightarrow V$ be a continuous $G$-equivariant map such that for all $t \in \mathbb{R}, f(t, 0)=0$. We are interested in solutions of the equation

$$
f(t, x)=0 .
$$

Clearly any pair $(t, 0)$ satisfies (46), thus we will call them a trivial solutions to (46). All other solutions to (46) will be called nontrivial. We denote the set of all nontrivial solutions to (46) by $\mathscr{S}$, i.e.

$$
\mathscr{S}:=\left\{(t, x) \in \mathbb{R} \times \mathbb{R}^{n} \oplus V: f(t, x)=0 \text { and } x \neq 0\right\}
$$

Definition B.2. Let $\mathcal{C} \subset \mathscr{S}$ and $\mathscr{U} \subset \mathbb{R} \times V \rightarrow V$ be a $G$-invariant open subset. The set $\mathcal{C} \subset \mathscr{S}$ is called a branch of nontrivial solutions to (46) in $\mathscr{U}$ if $\overline{\mathcal{C}}$ is a connected component of $\overline{\mathscr{S}} \cap \overline{\mathscr{U}}$. Moreover, we say that the branch $\mathcal{C}$ bifurcates from a trivial solution $\left(t_{o}, 0\right)$ if $\left(t_{o}, 0\right) \in \overline{\mathcal{C}}$.

Theorem B.3. Let $V$ be an orthogonal $G$-representation and $\Omega \subset V$ be a $G$-invariant bounded open set. Assume that $f:[0,1] \times V \rightarrow V$ is a continuous $G$-equivariant map such that for every $t \in[0,1],\left(f_{t}, \Omega\right)$ is an admissible $G$-pair and $\operatorname{deg}_{G}\left(f_{0}, \Omega\right) \neq 0$. Then there exists a compact connected set $K_{o} \subset f^{-1}(0) \cap[0,1] \times \Omega$ such that

$$
K_{o} \cap(\{0\} \times \Omega) \neq \emptyset \neq K_{o} \cap(\{1\} \times \Omega) .
$$

Let us discuss the global bifurcation problem for (46). Under the assumption that the derivative $D_{x} f(t, 0)$ exists for all $t \in \mathbb{R}$ and the map $t \mapsto D_{x} f(t, 0)$ is continuous, one can easily show that if $\left(t_{o}, 0\right)$ is a bifurcation point for (46), then $D_{x} f\left(t_{o}, 0\right)$ is not an isomorphism. Denote the set of all $t \in \mathbb{R}$ such that $(t, 0)$ is a bifurcation point of [46] by $\mathscr{B}$ and put

$$
\Lambda:=\left\{t \in \mathbb{R}: \operatorname{det} D_{x} f(t, 0)=0\right\} .
$$

$\Lambda$ is called the set of critical points for (46) and we clearly have $\mathscr{B} \subset \Lambda$.

The following result is called Rabinowitz's Alternative: 
Theorem B.4. Suppose that $f: \mathbb{R} \oplus V \rightarrow V$ is a continuous $G$-equivariant map such that $f(t, 0)=0$ for all $t \in \mathbb{R}$ and $D_{x} f(t, 0)$ exists and is continuous with respect to $t \in \mathbb{R}$. We also assume that the set of critical points $\Lambda$ for (46) (given by (477)) is discrete, and consider an open bounded $G$-invariant set $\mathcal{U} \subset \mathbb{R} \oplus V$ such that $\left(t_{o}, 0\right) \in \mathcal{U}$ for some $t_{o} \in \Lambda$. For a connected component $\mathscr{C}$ of the set $\overline{\mathcal{U}} \cap \overline{\mathscr{S}}$ such that $\left(t_{o}, 0\right) \in \mathscr{C}$ we have the following alternative.

(i) either $\mathscr{C} \cap \partial U \neq \emptyset$,

(ii) or $\mathscr{C} \cap(\Lambda \times\{0\})=\left\{\left(t_{1}, 0\right),\left(t_{2}, 0\right), \ldots,\left(t_{n}, 0\right)\right\}$ for some $n \in \mathbb{N}$ (here $t_{j} \neq t_{k}$ for $j \neq k$ ) and

$$
\sum_{k=1}^{n} \omega_{G}\left(t_{k}\right)=0 .
$$

\section{References}

[1] A. Ambrosetti and G. Mancini. Solutions of minimal period for a class of convex Hamiltonian systems. Math. Ann. 255 (1981), 405-421.

[2] Z. Balanov, W. Krawcewicz and H. Steinlein, Applied Equivariant Degree. AIMS Series on Differential Equations \& Dynamical Systems, Vol. 1, 2006.

[3] D. Birkoff and D.C.Lewis. On the periodic motions near a given periodic motion of a dynamical system. Ann. Math. Pure Appl. 12(1933), 117-133.

[4] A.Boscaggin and F. Zanolin. Subharmonic solutions for nonlinear second order equations in presence of lower and upper solutions. DCDS 33 (2013), 89-110.

[5] Z. Cheng and J. Ren. Periodic and subharmonic solutions for Duffing equation with singularity. DCDS 32 (2012), 1557-1574.

[6] C. Conley and E. Zehnder. Subharmonic solutions and Morse theory. Physica A 124 (1984), 649-658.

[7] T. Ding and F. Zanolin. Subharmonic solutions of second order nonlinear equations: a time-map approach. Nonlinear Analysis TYMA, 20 (5) (1993), 509-532.

[8] I. Ekeland and H. Hofer. Subharmonics for convex nonautonomous Hamiltonian systems. Comm. Pure Appl. Math. 40 (1987), 1-36.

[9] G. Feltrin. Positive solutions to superlinear ODEs with indefinite weight. DCDS S 11 (2018), 257-277.

[10] A. Fonda and A.C. Lazer. Subharmonic solutions of conservative systems with nonconvex potentials. Proc. Amer. Math. Soc. 115 (1992), 183-190.

[11] A. Fonda, M. Ramos and M. Willem. Subharmonic solutions for second order differential equations. Topol. Meth. Nonlinear Anal. 1 (1993), 49-66.

[12] A. Fonda and M. Willem. Subharmonic oscillations of forced pendulum type equations. J. Differential Equations 81 (1989), 215-220.

[13] E. Gaines and J. Mawhin. Coincidence Degree and Nonlinear Differential Equations. Vol. 568, Springer, 2006.

[14] F. Kong. Subharmonic solutions with prescribed minimal period of a forced pendulum equation with impulses. Acta App. Math. 158 (2018), 125-137.

[15] P. Martinez-Amores, J. Mawhin, R. Ortega and M. Willem. Generic results for the existence of nondegenerate periodic solutions of some differential systems with periodic nonlinearities. J. Differential Equations 91 (1991), 138-148.

[16] J. Mawhin. Topological Degree Methods in Nonlinear Boundary Value Problems, Amer. Math. Soc. 40(1979). 
[17] J. Mawhin and M. Willem. Multiple solutions of the periodic boundary value problem for some forced pendulum-type equations. J. Differential Equations 52 (1984), 264-287.

[18] J. Mawhin and M. Willem. Critical Point Theory and Periodic Solutions of Hamiltonian Systems. Springer, Berlin, 1989.

[19] R. Michalek and G. Tarantello. Subharmonic solutions with prescribed minimal period for nonautonomous Hamiltonian systems. J. Differential Equations 72 (1988), 28-55.

[20] D.C. Offin. Subharmonic solutions for forced pendulum type equations. Differential and Integral Equations 3 (1990), 965-972.

[21] Z. Opial. Sur les solutions périodiques de l'équation differentielle $x^{\prime \prime}+g(x)=p(t)$, Bull. Acad. Pol. Sci., Sér. Sci Math. 8 (1960), 151-156.

[22] P.H. Rabinowitz. Periodic solutions of Hamiltonian systems, Comm. Pure Appl. Math. 31 (1978), 157-184.

[23] P.H. Rabinowitz. On subharmonic solutions of Hamiltonian systems. Comm. Pure Appl. Math. 33 (1980), 609-633.

[24] P.H. Rabinowitz. On a class of functionals invariant under a $\mathbb{Z}_{n}$ action, Trans. Amer. Math. Soc. 310(1988), 303-311.

[25] P.H. Rabinowitz. Heteroclinics for a reversible Hamiltonian system. Ergod. Theoret. Dyn. System 14(1994), 817-829.

[26] E. Serra and M. Tarallo. A reduction method for periodic solutions of second order subquadratic equations. Adv. Differential Equations 3 (1998), 199-226.

[27] E. Serra, M. Tarallo and S. Terracini. On the structure of the solution set of forced pendulum-type equations. J. Differential Equations 131 (1996), 189-208.

[28] X. Wang and A. Qian. Existence and multiplicity of periodic solutions and subharmonic solutions for a class of elliptic equations. J. Nonlinear Sci. App. 10 (2017), 6229-6245.

[29] M. Willem. Perturbations of nondegenerate periodic orbits of Hamiltonian systems, Periodic Solutions of Hamiltonian Systems and Related Topics. Springer, Dordrecht, 1987, 261-265.

[30] R.S. Zounes and R.H. Rand. Subharmonic resonance in non-linear Mathieu equation. Int. J. Non-linear Mechanics 37 (2002), 43-73. 